\title{
Parimutuel Betting under Asymmetric Information *
}

\author{
Frédéric KoessleR ${ }^{\dagger} \quad$ Anthony ZIEGELMEYeR ${ }^{\ddagger}$
}

First Draft: October 2001

This Draft: June 7, 2002

\begin{abstract}
This paper examines a game-theoretical model of parimutuel betting markets with asymmetric information. Generically, all Bayesian-Nash equilibria of the simultaneous game are characterized depending on the number of bettors and the quality of their private information. An equilibrium where all bettors use their private information always exists and becomes unique as the number of bettors increases. On the contrary, in a sequential framework, a perfectly revealing equilibrium does not always exist and disappears as the number of bettors increases. Bettors arbitrate between following their private signal, following the choices of previous bettors, and betting against the trend. On average, information is well aggregated by the market, but extreme effects based on herd and contrarian behavior occur in identifiable states of the world, leading to significant mispricing.
\end{abstract}

KEYwORDS: Parimutuel betting; Asymmetric information; Information aggregation; Herd behavior; Contrarian behavior.

JEL Classification: C72; D82.

\section{Introduction}

In this paper we propose a game-theoretical analysis of parimutuel betting markets with asymmetric information. Such markets are characterized by well defined interactive decision situations which possess several features of commonly analyzed games. First, they are anonymous games in the sense that a player's payoff only depends on the distribution of strategies over the set of players rather than on other players' identities. Second, they are characterized by negative payoff externalities as the payoff of each player decreases with the number of other players who bet on the same option. Third, they are "almost" $n$-person zero-sum games since, except in the case where all bettors choose the incorrect option, the return to each individual is a fractional proportion of the entire amount wagered on the market. Finally, they are characterized by a well-defined termination point at which the value of each bet becomes certain. In consequence, the analysis of parimutuel markets can provide a clear view of pricing issues and information aggregation which are more complicated elsewhere.

The origin of parimutuel markets lies in horse-race betting. In this paper we consider parimutuel gambling systems used by racetracks in North America, France, Hong Kong, and

\footnotetext{
${ }^{*}$ We thank Charles Noussair, Patrick Roger and Gisèle Umbhauer for helpful comments and enriching discussions. We are also grateful to Han Guoniu for helping us proving some results of this paper.

${ }^{\dagger}$ BETA, Université Louis Pasteur, 61 Avenue de la Forêt-Noire, F-67085 Strasbourg Cedex (France). Email: koessler@cournot.u-strasbg.fr

${ }^{\ddagger}$ Max Planck Institute for Research into Economic Systems, Strategic Interaction Group, Jena (Germany). Email: ziegelmeyer@mpiew-jena.mpg.de
} 
Japan, where individuals directly place bets on horses. In such systems, if the horse chosen by an individual wins, then his investment yields returns (calculated through final betting odds) that are decreasing with the proportion of bettors who bet on the same horse. Besides, the proportion of the money in the win pool that is bet on any given horse is interpreted as the subjective probability that this horse will win the race and it corresponds to the implicit price of a one unit of money bet. Several theoretical and empirical studies in economics have investigated the features of parimutuel betting markets and have pointed out their particular relevance for the analysis of decision making under risk and for the analysis of market efficiency. ${ }^{1}$

As a whole, the few existing theoretical researches on racetrack parimutuel betting have focused on games with symmetric information, either by considering bettors with homogeneous beliefs (Chadha and Quandt, 1996; Feenay and King, 2001), or by introducing uninformed bettors modeled as "irrational" noise bettors (Hurley and McDonough, 1995; Terrell and Farmer, 1996; Koessler, Ziegelmeyer, and Broihanne, 2002), or by endowing bettors with inconsistent beliefs (Watanabe, Nonoyama, and Mori, 1994; Watanabe, 1997). Contrary to those previous contributions, we propose to explore a game-theoretical model of parimutuel betting with asymmetric information and consistent beliefs. That is, we consider strategic parimutuel betting markets where differences in beliefs are due only to differences in information, and are not arbitrary unexplained differences in opinions. Those differences in information may be due, e.g., to the dispersion of knowledge concerning the intrinsic ability of each horse, the condition of the track, the skill of each jockey, horses' performances in previous races, etc. In such a setting, we especially wish to analyze the relative effectiveness of sequential betting vis-à-vis simultaneous betting at aggregating information from diverse sources.

To begin with, we consider as a benchmark framework the simultaneous move version of the game. Generically (for almost all bettors' qualities of information), we characterize the set of pure strategy Bayesian-Nash equilibria according to the number of bettors and the quality of their private information. In particular, we show that a "fully informative" equilibrium where all bettors use their private information always exists. Asymmetric equilibria where some players bet non-informatively on the same horse whatever their private signal also exist. Though such a multiplicity of equilibria raises selection questions, we show that such a selection is needless for a large number of bettors since asymmetric equilibria vanish when the number of bettors increases. More precisely, when the number of bettors increases, asymmetric equilibria exist only for small intervals of the players' information quality and they are characterized by an increasing number of informative bets. Consequently, odds against each horse perfectly reflect all private information in large wagering markets.

Next, we investigate sequential betting in an attempt to capture the strategic behavior of static (simultaneous) betting games, the social learning of sequential trade models, and signaling behavior. In such a setting, bets, in themselves, could reveal the underlying information and so affect the behavior of latter bettors. Therefore, the effect of information on odds takes an added dimension as the possibility of multiple rounds of bets is considered. We first show that a perfectly revealing equilibrium can fail to exist, and will typically disappear when the number of bettors increases. Intuitively, this failure might be related to the fact that bettors exhibit herd behavior. Such behavior arises if later decision makers choose to blindly imitate their predecessors' choice without heed to their own private information. However, as in most multi-agent sequential trade models with asymmetric information, complete imitation does not hold in the long-run since it dilutes the profit of early agents. ${ }^{2}$ Actually, we show that bettors arbitrate between following their private signal, following the choices of previous

\footnotetext{
${ }^{1}$ A detailed account of this recently developed literature may be found in Sauer (1998) and Vaughan Williams (1999).

${ }^{2}$ For a survey on sequential trade models with asymmetric information see, e.g., Brunnermeier (2001).
} 
bettors (herd behavior), and betting against the trend (contrarian behavior). These choices depend on the quality of bettors' information, on the information acquired from past bets, and on the way players forecast future participants' information so as to predict future prices (odds).

Unfortunately, it is intractable to provide a complete characterization of the set of equilibria for an arbitrary number of players in the sequential betting game. Indeed, bettors' decision rules involve both backward (learning) and forward (signaling) behaviors which prevent the computation of rational strategies due to the intractability of a full lookahead in the game tree. Instead, we characterize conditions for some classes of equilibria to exist, we examine the set of equilibria with two and three bettors and, thereafter, we consider less sophisticated bettors (called "myopic" bettors) who are not able to anticipate the future fluctuation of betting odds. That is, myopic bettors perform no lookahead in the sense that they consider the current odds as the final odds. As with fully rational bettors, it is shown that odds adjustments do not prevent herd and contrarian behavior because they do not integrate all information available in the history of bets. Nevertheless, we show that, on average, information is well aggregated even if herd behavior leads to extreme effects in some states of the world.

It is worth mentioning that the model and results presented in this paper can be applied to a variety of problems of investment decisions in which the payoff of each agent is decreasing with the number of agents who make the same decision and where individuals are asymmetrically informed about the return of each alternative. In particular, our study can be applied to models of congestion where the utility of a product like a transportation device, a firm's location, or a visit of a public event, decreases with the quantity of users. For example, as suggested by Feenay and King (2001), bettors in parimutuel markets may be assimilated to investors who have to choose between several towns in which to locate similar retail stores. Introducing asymmetric information, as is made in the present paper, allows one to analyze a situation in which investors are privately informed about, e.g., the state of demand in each town.

The paper proceeds as follows. Section 2 lays out the framework and basic model of parimutuel betting used throughout the paper. In Section 3, we cover the results concerning the set of all pure strategy Bayesian equilibria in the benchmark simultaneous move betting game. Section 4 investigates the sequential move version of the game. Equilibrium outcomes and information aggregation with myopic bettors are analyzed in Section 5. We give concluding remarks and we discuss the relationship with previous studies in Section 6. All proofs can be found in the Appendix.

\section{A Model of Parimutuel Betting}

In this section we present a general framework of racetrack parimutuel betting. As most theoretical studies in this literature, we consider a horse race where there are only two horses. Additionally, we do not allow bettors to withdraw from betting and we assume that each bettor is only able to bet one unit of money. Said differently, bettors must spend on the race their entire endowment of one unit of money. If bettors were able to drop from betting, then a no-trade result would apply and the only equilibrium would be the trivial equilibrium where all bettors refrain from betting. ${ }^{3}$ Finally, we consider a finite set of asymmetrically informed bettors. All of them are assumed to integrate the direct consequences of their actions on the odds of the gamble, and they are endowed with beliefs about the state of Nature obtained from their signals and from the information contained in the choices of their predecessors.

\footnotetext{
${ }^{3} \mathrm{~A}$ complementary analysis to the one presented in this paper would be to investigate a model with withdrawing possibilities by adding players who bet for exogenous reasons.
} 
Additionally, with the exception of Section 5 where we consider myopic agents, bettors are fully rational players in the sense that they incorporate in their optimization problem their beliefs about successors' strategies and beliefs. In that way, they take into account their full impact on the equilibrium betting odds.

Independently of its temporal structure, the parimutuel betting market can be modeled as follows. The two horses are called $A$ and $B$. There is a finite set $N=\{1, \ldots, n\}$ of risk neutral bettors. Each bettor $i \in N$ independently chooses to bet one unit of money on a horse $s_{i} \in S_{i} \equiv\{A, B\}$. For any vector of bets $s=\left(s_{1}, \ldots, s_{n}\right) \in S=\prod_{i \in N} S_{i}$ and any horse $H \in\{A, B\}$, let $H(s)=\left\{i \in N: s_{i}=H\right\}$ be the set of bettors who bet on horse $H$, let $h(s)=|H(s)|$ be the number of bettors who bet on horse $H$, et let $\bar{H}$ be the horse other than $H$. The odds against horse $H$, which is given by the total number of bets on horse $\bar{H}$ divided by the total number of bets on horse $H$, is denoted by

$$
O_{H}(s)=\frac{n-h(s)}{h(s)} .
$$

We assume that bettors have a common prior belief on the payoff-relevant state space (or set of states of Nature) $\Theta=\left\{\theta_{A}, \theta_{B}\right\}$, where $\theta_{A}$ stands for "horse $A$ wins" and $\theta_{B}$ stands for "horse $B$ wins". For simplicity, we consider a flat prior belief: $\operatorname{Pr}\left(\theta_{A}\right)=\operatorname{Pr}\left(\theta_{B}\right)=1 / 2$. The results would be essentially the same with unequal prior winning chances. ${ }^{4}$

If bettor $i$ bets on the winning horse, then his payoff is normalized to the return of this horse, which is equal to its odds plus 1 . Otherwise, bettor $i$ receives 0 payoff. Hence, the payoff of each bettor depends on his own choice, on alternatives chosen by other bettors, and on the actual state of Nature. For example, odds of 4 to 1 laid against a horse implies a payoff to a successful bet of four units of money, plus the stake returned (which is equal to one unit of money here); on the contrary, an unsuccessful bet loses the stake. Formally, each bettor $i \in N$ has a utility function $u_{i}: S \times \Theta \rightarrow \mathbb{R}$. Given a pair of bettors' actions and a state of Nature, $(s, \theta) \in S \times \Theta$, the utility derived by bettor $i$ is given by $u_{i}(s, \theta)$. For all $H \in\{A, B\}$, bettor $i$ 's utility function satisfies

$$
u_{i}(s, \theta)= \begin{cases}O_{H}(s)+1=\frac{n}{h(s)} & \text { if } s_{i}=H \text { and } \theta=\theta_{H} \\ 0 & \text { if } s_{i}=H \text { and } \theta \neq \theta_{H} .\end{cases}
$$

Before taking his decision, and in addition to the possible history of choices he has observed, each bettor $i \in N$ gets a private signal $q_{i} \in Q_{i} \equiv\left\{q^{A}, q^{B}\right\}$ that is correlated to the true state of Nature. Conditionally on the state of Nature, bettors' signals are i.i.d. and satisfy

$$
\begin{aligned}
& \operatorname{Pr}\left(q_{i}=q^{A} \mid \theta_{A}, q_{j}\right)=\operatorname{Pr}\left(q_{i}=q^{A} \mid \theta_{A}\right)=\pi>1 / 2 \\
& \operatorname{Pr}\left(q_{i}=q^{A} \mid \theta_{B}, q_{j}\right)=\operatorname{Pr}\left(q_{i}=q^{A} \mid \theta_{B}\right)=1-\pi,
\end{aligned}
$$

for all $i, j \in N, i \neq j$. Hence, once a bettor $i \in N$ has received a signal $q_{i} \in Q_{i}$, his beliefs about the states of Nature are given by

$$
\begin{aligned}
& \operatorname{Pr}\left(\theta_{A} \mid q^{A}\right)=\operatorname{Pr}\left(\theta_{B} \mid q^{B}\right)=\pi \\
& \operatorname{Pr}\left(\theta_{A} \mid q^{B}\right)=\operatorname{Pr}\left(\theta_{B} \mid q^{A}\right)=1-\pi .
\end{aligned}
$$

The parameter $\pi$ characterizes bettors' quality or precision of information. The larger is $\pi$, the larger is the precision of bettors' signals.

\footnotetext{
${ }^{4}$ For example, if bets are made simultaneously and if we consider different prior beliefs for horses $A$ and $B$, then, in asymmetric equilibria, the number of non-informative bets on each horse are not necessarily the same. Actually, it can be shown that in such equilibria, when they exist, the number of unconditional bets on horse $A$ are increasing with the prior probability of horse $A$, and inversely for horse $B$. We do not consider those generalizations since they introduce integer problems which complicate the exposition without substantially modifying the results.
} 


\section{Simultaneous Betting}

To avoid the complexities associated with social learning and signaling of sequential games, we first analyze the simultaneous parimutuel betting game. In such a setting, a bettor's strategy is a rule of action which maps each realization of his signal to one of two actions: to bet on horse $A$, or to bet on horse $B$. More precisely, a (pure) strategy of bettor $i$ is a mapping $\sigma_{i}: Q_{i} \rightarrow S_{i}$. The set of strategies of each bettor $i$ is denoted by $\Sigma_{i}$, and the set of strategy profiles is denoted by $\Sigma=\prod_{i \in N} \Sigma_{i}$. Bettor $i$ 's belief that the winning horse is horse $H \in\{A, B\}$ and that other bettors receive the vector of signals $q_{-i} \in Q_{-i}$ is given by

$$
\operatorname{Pr}\left(\theta_{H}, q_{-i} \mid q_{i}\right)=\operatorname{Pr}\left(q_{-i} \mid \theta_{H}\right) \operatorname{Pr}\left(\theta_{H} \mid q_{i}\right)=\left(\prod_{j \neq i} \operatorname{Pr}\left(q_{j} \mid \theta_{H}\right)\right) \operatorname{Pr}\left(\theta_{H} \mid q_{i}\right) .
$$

Thus, using Equations (1) and (2), bettor $i$ 's expected utility given others' strategies $\sigma_{-i}$ when he bets on horse $H$ and receives the signal $q_{i}$ can be written

$$
U_{i}\left(H, \sigma_{-i} \mid q_{i}\right)=\operatorname{Pr}\left(\theta_{H} \mid q_{i}\right) \sum_{q_{-i} \in Q_{-i}}\left(\prod_{j \neq i} \operatorname{Pr}\left(q_{j} \mid \theta_{H}\right)\right) \frac{n}{h\left(H, \sigma_{-i}\left(q_{-i}\right)\right)} .
$$

As usual, in a Bayesian-Nash equilibrium the action prescribed by each bettor's strategy maximizes his expected payoff conditional on his signal given others' strategies. More precisely, a Bayesian-Nash equilibrium, or simply equilibrium, is a profile of strategies $\sigma$ such that for all $i \in N, q_{i} \in Q_{i}$, and $s_{i} \in S_{i}$ we have $U_{i}\left(\sigma_{i}\left(q_{i}\right), \sigma_{-i} \mid q_{i}\right) \geq U_{i}\left(s_{i}, \sigma_{-i} \mid q_{i}\right) .^{5}$ The following proposition establishes that there is no equilibrium of the simultaneous move game where a bettor systematically bets against his private signal.

Proposition 1 For any number of bettors, $n$, and any quality of information, $\pi$, the strategy which involves some bettor $i \in N$ in betting against his private signal, i.e., $\sigma_{i}\left(q^{H}\right)=\bar{H}$ for all $H \in\{A, B\}$, is strictly dominated.

On the contrary, a natural and intuitive strategy for a bettor is simply to follow his private signal, i.e., to bet on horse $A$ if he receives the signal $q^{A}$ and to bet on horse $B$ if he receives the signal $q^{B}$. In the following proposition we show that there is always an equilibrium where all bettors use this "informative" strategy, and this equilibrium is the unique symmetric one. We call this equilibrium the fully informative equilibrium. In such an equilibrium, betting odds perfectly reflect all private information.

Proposition 2 For any number of bettors, $n$, and any quality of information, $\pi$, there exists an equilibrium where every bettor follows his private signal, i.e., $\sigma_{i}\left(q^{H}\right)=H$ for all $H \in$ $\{A, B\}$ and $i \in N$. Additionally, this equilibrium is the unique symmetric equilibrium.

Now, we investigate the possibility of asymmetric equilibria, i.e., equilibria in which bettors use different strategies. We first characterize conditions under which no bettor uses his private information. An equilibrium in which no bettor uses his private information is called a noninformative equilibrium. For any non-negative integers $j$ and $k$, the binomial coefficient is denoted by $C_{k}^{j} \equiv \frac{k !}{j !(k-j) !}$.

\footnotetext{
${ }^{5}$ Since we deal in this paper only with pure strategy equilibria, no confusion shall arise when we write "equilibrium" instead of "pure strategy equilibrium". To simplify the exposition, we ignore indifference cases throughout the paper when they are due to non-generic values of $\pi$.
} 
Proposition 3 There exists a non-informative equilibrium where $a \in\{0,1, \ldots, n\}$ bettors always bet on horse $A$ and $b=n-a$ bettors always bet on horse $B$ if and only if $n$ is an even number, $a=b=n / 2$, and $\pi \leq \frac{n+2}{2 n+2}$. If such an equilibrium exists, then there are exactly $C_{n}^{n / 2}$ non-informative equilibria.

It is interesting to note that $\lim _{n \rightarrow \infty} \frac{n+2}{2 n+2}=1 / 2$. Hence, for a large number of bettors, noninformative equilibria exist only for very low qualities of private information. As shown below, the same phenomenon arises for all other asymmetric equilibria, namely partially informative ones. More precisely, a partially informative equilibrium is an equilibrium in which some bettors bet informationally and each other bettor bets on a given horse whatever his private signal. A necessary and sufficient condition for such an equilibrium to exist is given by the following proposition.

Proposition 4 There exists a partially informative equilibrium where exactly $k$ bettors always follow their private signal if and only if $n-k$ is an even number, $\frac{n-k}{2}$ bettors always bet on horse $A, \frac{n-k}{2}$ bettors always bet on horse $B$, and the following inequality is satisfied:

$$
\frac{\pi}{1-\pi} \leq \frac{\sum_{j=0}^{k} \frac{C_{k}^{j} \pi^{j}(1-\pi)^{k-j}}{\frac{n-k}{2}+j}}{\sum_{j=0}^{k} \frac{C_{k}^{j} \pi^{j}(1-\pi)^{k-j}}{\frac{n-k}{2}+1+j}}=\frac{\sum_{j=0}^{k} \frac{\left(\frac{\pi}{1-\pi}\right)^{j}}{j !(k-j) !\left(\frac{n-k}{2}+j\right)}}{\sum_{j=0}^{k} \frac{\left(\frac{\pi}{1-\pi}\right)^{j}}{j !(k-j) !\left(\frac{n-k}{2}+j+1\right)}} .
$$

If such an equilibrium exists, then there are exactly $C_{n}^{k} \times C_{n-k}^{\frac{n-k}{2}}$ partially informative equilibria with $k$ informative bets.

A partially informative equilibrium with exactly $k$ informative bets is called a $k$-informative equilibrium. Note that the case $k=n$ corresponds to the fully informative equilibrium, which always exists from Proposition 2. If $k=0$, we get the non-informative equilibrium of Proposition 3.

Example 1 Consider $k=1$ bettor who follows his signal and consider any odd number of bettors, $n$. In that case we have $a=b=(n-1) / 2$, and Condition (3) is equivalent to

$$
\frac{\pi}{1-\pi} \leq \frac{\frac{1-\pi}{a}+\frac{\pi}{a+1}}{\frac{1-\pi}{a+1}+\frac{\pi}{a+2}}
$$

i.e., $\pi \leq \frac{1}{1+\sqrt{\frac{a}{2+a}}}=\frac{1}{1+\sqrt{\frac{n-1}{n+3}}}$. We remark that $\lim _{n \rightarrow \infty} \frac{1}{1+\sqrt{\frac{n-1}{n+3}}}=1 / 2$, which means that the condition for the existence of a 1-informative equilibrium becomes stronger as the number of bettors increases. We will generalize this result to all partially informative equilibria.

Solving Condition (3) for $\pi$ with arbitrarily high values of $k$ should not be conceivable. However, we provide in the following lines interesting properties of such a solution. We first show that values of $\pi$ satisfying the condition for a $k$-informative equilibrium to exist belong to an interval $[1 / 2, \pi(k, n)]$, where $\pi(k, n)<1$. To do this, we begin to give in the following lemma a simpler formulation of Condition (3), and we present in subsequent lemmas useful properties of this new formulation.

Lemma 1 Condition (3) is satisfied if and only if $g(k, n, \pi) \leq 0$, where

$$
g(k, n, \pi)=\sum_{j=0}^{k+1}\left(\frac{\pi}{1-\pi}\right)^{j} C_{k+1}^{j} \frac{k-2 j+1}{k-2 j-n} .
$$


Lemma 2 For all $n \geq 2$ and $k<n$, there exists $\left.\pi, \pi^{\prime} \in\right] 1 / 2,1[$ such that $g(k, n, \pi)<0$ and $g\left(k, n, \pi^{\prime}\right)>0$.

Lemma 3 For all $n \geq 2$ and $k<n, g(k, n, \cdot)$ is strictly increasing with $\pi$ on the interval ] $1 / 2,1[$.

From those three lemmas we know that the polynomial in $\pi$ induced by Equation (3) has one and only one real root on the interval $] 1 / 2,1[$. Actually, we have more: for sufficiently small qualities of information, there exist partially informative equilibria as described in Proposition 4. More precisely:

Proposition 5 For all $n \geq 2$ and $k<n$ such that $n-k$ is an even number, there exists $\pi(k, n) \in] 1 / 2,1[$ such that Condition (3) is satisfied if and only if $\pi \leq \pi(k, n)$.

We now investigate the properties of $\pi(k, n)$, where the value of $\pi(k, n)$ corresponds to the maximum quality of information for a $k$-informative equilibrium to exist. According to the next conjecture, for any number $n$ of bettors, conditions for the existence of a partially informative equilibrium are weaker as the number of informative bets, $k$, increases. ${ }^{6}$

Conjecture 1 For all $n \geq 2$ and $k \in\{1, \ldots, n-2\}$, we have $\pi(k, n)>\pi(k-1, n)$.

The next conjecture presents an additional useful property of the function $\pi(k, n)$. It is argued that $\pi(n-2, n)<\pi(n-3, n-1)$ for any number $n$ of bettors. Said differently, the maximum quality of information allowing the existence of a $(n-2)$-informative equilibrium (one non-informative bet on each horse) is decreasing in $n$.

Conjecture 2 For all $n \geq 3$ we have $\pi(n-2, n)<\pi(n-3, n-1)$.

From those properties we can immediately establish the two following corollaries. ${ }^{7}$

Corollary 1 For all $n \geq 3$ we have $\pi(k, n)<\pi(k, n-1)$ and $\lim _{n \rightarrow \infty} \pi(k, n)=1 / 2$, for all $k \in\{0,1, \ldots, n-2\}$.

Corollary 2 Let $n \geq 2$ and $k \in\{2, \ldots, n-2\}$ such that $n-k$ is an even number.

- The fully informative equilibrium is unique if and only if $\pi>\pi(n-2, n)$;

- If $\pi(k-2, n)<\pi \leq \pi(k, n)$, then the less informative equilibria are the $k$-informative ones and there exist $l$-informative equilibria for all $l \in\{k, \ldots, n-2\}$ such that $n-l$ is an even number.

In particular, since $\pi(0,2)=2 / 3>\pi(k, n)$ for all $k>0$ and $n>2$, if $\pi>2 / 3$, then for any number of bettors, the unique equilibrium is the fully informative one.

\section{Sequential Betting}

While in most equilibria of the simultaneous betting game odds against horses reflect all players' private information, bettors cannot use this publicly revealed information. Hence, all dimensions of information aggregation can only be examined in a sequential framework. In this respect, we explore in this section the predictions of our model under a more general assumption regarding observability of actions by letting bets chosen by any player to be observable by the rest of the market.

\footnotetext{
${ }^{6}$ Conjectures 1 and 2 have been checked numerically up to $n=40$ bettors for all possible values of $k$. The Mathematica code is available from the authors upon request.

${ }^{7}$ Of course, the validity of these two corollaries depends on the validity of Conjectures 1 and 2 . No other results in the paper depend on those conjectures.
} 


\subsection{Strategies, Beliefs, and Expected Utilities}

At each stage in the sequence, bettors can observe the bets cast by preceding bettors, but not the signals that those earlier bettors received. A history of $k$ decisions is denoted by $s^{k} \in S^{k}=$ $\prod_{j=1}^{k}\{A, B\}$. When bettor $k+1$ takes a decision, he observes a history $s^{k}$, i.e., he observes the decisions taken by bettors $1, \ldots, k$. For all $H \in\{A, B\}$, let $H\left(s^{k}\right)=\left\{i \in\{1, \ldots, k\}: s_{i}=H\right\}$ be the set of bettors up to period $k$ who chose horse $H$, and let $h\left(s^{k}\right)=\left|H\left(s^{k}\right)\right|$ be the associated number of bets on horse $H$.

A (pure) strategy of bettor $i$ is a function $\sigma_{i}: S^{i-1} \times Q_{i} \rightarrow S_{i}$. Hence, $\sigma_{i}\left(s^{i-1} ; q_{i}\right) \in S_{i}$ is the choice of bettor $i$ with signal $q_{i} \in Q_{i}=\left\{q^{A}, q^{B}\right\}$ when he has observed the history $s^{i-1} \in S^{i-1}$. A profile of strategies is denoted by $\sigma=\left(\sigma_{1}, \ldots, \sigma_{n}\right) \in \Sigma=\prod_{i \in N} \Sigma_{i}$. Denote respectively by $q_{i^{+}}=\left(q_{j}\right)_{j>i}$ and $\sigma_{i^{+}}=\left(\sigma_{j}\right)_{j>i}$ the signals and strategies of the successors of bettor $i$, and let $Q_{i^{+}}=\prod_{j>i} Q_{j}$ and $\Sigma_{i^{+}}=\prod_{j>i} \Sigma_{j}$. Let $s\left(\sigma_{i^{+}} \mid s^{i} ; q_{i^{+}}\right)$be the final history reached according to the profile of strategies $\sigma_{i^{+}} \in \Sigma_{i^{+}}$given the history $s^{i} \in S^{i}$ and the profile of signals $q_{i^{+}} \in Q_{i^{+}}$. Let $s(\sigma \mid q)=s(\sigma \mid \emptyset ; q)$ be the final history reached according to the profile of strategies $\sigma \in \Sigma$ given the profile of signals $q \in Q$, and let $s\left(\sigma_{n^{+}} \mid s^{n} ; q_{n^{+}}\right)=s^{n}$ (with some abuse of notation since $\sigma_{n^{+}}$and $q_{n^{+}}$are undetermined objects). Bettor $i$ 's belief about the state of Nature $\theta \in \Theta$ when he has received the signal $q_{i} \in Q_{i}$ and has observed the history $s^{i-1} \in S^{i-1}$ is denoted by $\mu_{i}\left(\theta \mid s^{i-1} ; q_{i}\right)$. The conditional independence of the signals gives, for $H \in\{A, B\}$,

$$
\operatorname{Pr}\left(\theta_{H}, q_{i^{+}} \mid s^{i-1}, q_{i}\right)=\operatorname{Pr}\left(q_{i^{+}} \mid \theta_{H}\right) \mu_{i}\left(\theta_{H} \mid s^{i-1} ; q_{i}\right) .
$$

Given a profile of strategies $\sigma_{i^{+}} \in \Sigma_{i^{+}}$, and bettor $i$ 's belief function $\mu_{i}$, bettor $i$ 's expected utility is obtained from Equation (4) and from bettors' utility function (see Equation (1) on page 4):

$$
U_{i}\left(H, \sigma_{i^{+}} \mid s^{i-1} ; q_{i}\right)=\mu_{i}\left(\theta_{H} \mid s^{i-1} ; q_{i}\right) E\left(\frac{n}{h\left(s\left(\sigma_{i^{+}} \mid s^{i-1}, H ; q_{i^{+}}\right)\right)} \mid \theta_{H}\right),
$$

where $E\left(\frac{n}{h\left(s\left(\sigma_{i^{+}} \mid s^{i-1}, H ; q_{i}\right)\right)} \mid \theta_{H}\right)=\sum_{q_{i^{+}} \in Q_{i^{+}}} \operatorname{Pr}\left(q_{i^{+}} \mid \theta_{H}\right) \frac{n}{h\left(s\left(\sigma_{i^{+}} \mid s^{i-1}, H ; q_{i^{+}}\right)\right)}$is the expected final gross return of horse $H$ for bettor $i$, where the final gross return of horse $H$ is equal to the final odds against horse $H$ plus one.

\subsection{Rational Strategies and Consistent Beliefs}

As usual, a pair $(\sigma, \mu)$ is a perfect Bayesian equilibrium if $\mu$ is obtained by Bayes' rule whenever possible (weak consistency), and $\sigma$ satisfies sequential rationality. Formally, a pair $(\sigma, \mu)$ is sequentially rational if for all $i \in N, q_{i} \in Q_{i}$, and $s^{i-1} \in S^{i-1}$ we have $U_{i}\left(\sigma_{i}\left(s^{i-1} ; q_{i}\right), \sigma_{i^{+}}\right.$। $\left.s^{i-1} ; q_{i}\right) \geq U_{i}\left(s_{i}, \sigma_{i^{+}} \mid s^{i-1} ; q_{i}\right)$, for all $s_{i} \in S_{i}$. For all $j \in N$, let $J_{j}\left(s_{j} \mid s^{j-1}\right) \equiv\left\{q_{j} \in Q_{j}\right.$ : $\left.\sigma_{j}\left(s^{j-1} ; q_{j}\right)=s_{j}\right\}$ be the set of signals such that bettor $j$ plays $s_{j}$ after he has observed the history $s^{j-1} \in S^{j-1}$. If $J_{j}\left(s_{j} \mid s^{j-1}\right) \neq \emptyset$ for all $j<i$, then bettor $i$ can apply Bayes' rule: $\mu_{i}\left(\theta \mid s^{i-1} ; q_{i}\right)=\operatorname{Pr}\left(\theta \mid q_{i}, q_{j} \in J_{j}\left(s_{j} \mid s^{j-1}\right) \forall j<i\right)$. Hence, a pair $(\sigma, \mu)$ is weakly consistent if for all $i \in N, q_{i} \in Q_{i}$, and $s^{i-1} \in S^{i-1}$ we have

$$
\mu_{i}\left(\theta \mid s^{i-1}, q_{i}\right)=\frac{\operatorname{Pr}\left(q_{i} \mid \theta\right) \prod_{j<i} \sum_{q_{j} \in J_{j}\left(s_{j} \mid s^{j-1}\right)} \operatorname{Pr}\left(q_{j} \mid \theta\right)}{\sum_{\theta_{H} \in\left\{\theta_{A}, \theta_{B}\right\}} \operatorname{Pr}\left(q_{i} \mid \theta_{H}\right) \prod_{j<i} \sum_{q_{j} \in J_{j}\left(s_{j} \mid s^{j-1}\right)} \operatorname{Pr}\left(q_{j} \mid \theta_{H}\right)},
$$

whenever $J_{j}\left(s_{j} \mid s^{j-1}\right) \neq \emptyset$ for all $j<i$. The next lemma will be useful in several steps of our analysis:

Lemma 4 Bettor $n$ bets on horse $H \in\{A, B\}$ if and only if $\mu_{n}\left(\theta_{H} \mid s^{n-1} ; q_{n}\right) \geq \frac{h\left(s^{n-1}\right)+1}{n+1}$. 
In the next subsection we characterize the set of perfectly revealing equilibria. Contrary to the simultaneous setting we show that such equilibria do not always exist in a sequential betting market, and they disappear as the number of bettors increases.

\subsection{Perfectly Revealing Equilibrium}

At a perfectly revealing equilibrium, each bettor follows his own private signal whatever the history he has observed. Formally, an equilibrium $(\sigma, \mu)$ is said perfectly revealing if each bettor follows his private signal, i.e., $\sigma_{i}\left(s^{i-1} ; q^{H}\right)=H$ for all $i \in N, s^{i-1} \in S^{i-1}$, and $H \in\{A, B\}$. More generally, a profile of strategies $\left(\sigma_{i}\right)_{i<j}$ is said perfectly revealing if $\sigma_{i}\left(s^{i-1} ; q^{H}\right)=H$ for all $i<j, s^{i-1} \in S^{i-1}$, and $H \in\{A, B\}$. Perfectly revealing equilibria are "quite" easy to analyze because an individual's bet does not influence the actions of later bettors, given that they always follow their private signal. Therefore, there is no incentive to deviate from the equilibrium to change the bets of subsequent bettors because every information set in the game tree is reachable with positive probability via perfectly revealing strategies. The next lemma characterizes bettors' updated beliefs when previous bettors used perfectly revealing strategies.

Lemma 5 If the profile of strategies $\left(\sigma_{j}\right)_{j<i}$ is perfectly revealing, then bettor $i$ 's beliefs are given by

$$
\begin{aligned}
\mu_{i}\left(\theta_{H} \mid s^{i-1} ; q^{H}\right) & =\frac{\pi^{2 h+2-i}}{\pi^{2 h+2-i}+(1-\pi)^{2 h+2-i}}, \\
\text { and } \quad \mu_{i}\left(\theta_{H} \mid s^{i-1} ; q^{\bar{H}}\right) & =\frac{\pi^{2 h-i}}{\pi^{2 h-i}+(1-\pi)^{2 h-i}},
\end{aligned}
$$

for all $H \in\{A, B\}$ and $s^{i-1} \in S^{i-1}$, where $h=h\left(s^{i-1}\right)$.

We begin by noting in the following proposition that there is a range of parameter values for which perfectly revealing betting fails to be an equilibrium. More precisely, it is shown that if the quality of bettors' private information is not fairly balanced, then a perfectly revealing equilibrium does not exist. The intuition is the following. Consider the last bettor in the sequence (bettor $n$ ). If the quality of information is too low and the private signal of bettor $n$ is in accordance with previous decisions, then the majority of past bets on the favorite is not sufficiently convincing that the favorite will really win, and thus it is optimal for bettor $n$ to bet against the trend and his own private signal. Such a behavior is called a contrarian behavior as the agent ignores his private signal and bets against the current favorite. On the contrary, if the quality is too high, then previous bets provide strong evidence that the favorite will win, and thus bettor $n$ will not follow his signal if it favors the longshot. This behavior is called herd behavior as the agent follows the trend independently of his private signal. In this later case, the fact that previous bettors have bet on the same horse reveals relevant information about the winning chance of this horse (the favorite). Such public information may overwhelm the bettor's private assessment and may counterbalance the negative effect of the odds against the favorite (which is low), and thus may cause a bettor otherwise inclined to bet on the longshot to bet on the horse chosen by previous bettors.

Proposition 6 Let $n>2$. If $(\sigma, \mu)$ is a perfectly revealing equilibrium, then

$$
\frac{n^{1 /(n-2)}}{1+n^{1 /(n-2)}} \geq \pi \geq \frac{n^{1 / n}}{1+n^{1 / n}}
$$


This necessary condition for a perfectly revealing equilibrium to exist becomes more and more restrictive as the number of bettors, $n$, increases. In the following proposition we give necessary and sufficient conditions for a perfectly revealing equilibrium to exist.

Proposition 7 There exists a perfectly revealing equilibrium if and only if the following inequality is satisfied for all $i \in N$ and $a \in\{0,1,2, \ldots, i-1\}$ :

$$
\left(\frac{\pi}{1-\pi}\right)^{2 a+2-i} \geq \frac{\sum_{j=0}^{n-i} \frac{C_{n-i}^{j} \pi^{j}(1-\pi)^{n-i-j}}{i-a+j}}{\sum_{j=0}^{n-i} \frac{C_{n-i}^{j} \pi^{j}(1-\pi)^{n-i-j}}{a+1+j}} .
$$

For bettor 1 this condition is always satisfied, and for bettor $n$ it simplifies to

$$
\left(\frac{\pi}{1-\pi}\right)^{2 a+2-n} \geq \frac{\frac{1}{i-a}}{\frac{1}{a+1}}=\frac{a+1}{n-a},
$$

For any number of bettors between two and fifteen, the intervals of qualities of information $[\underline{\pi}(n), \bar{\pi}(n)]$ allowing a perfectly revealing equilibrium to exist are given in Figure 1 . Whatever the quality of information, $\pi$, there is no perfectly revealing equilibrium when the number of bettors exceeds eleven as $\underline{\pi}(n)>\bar{\pi}(n)$ when $n>11$.

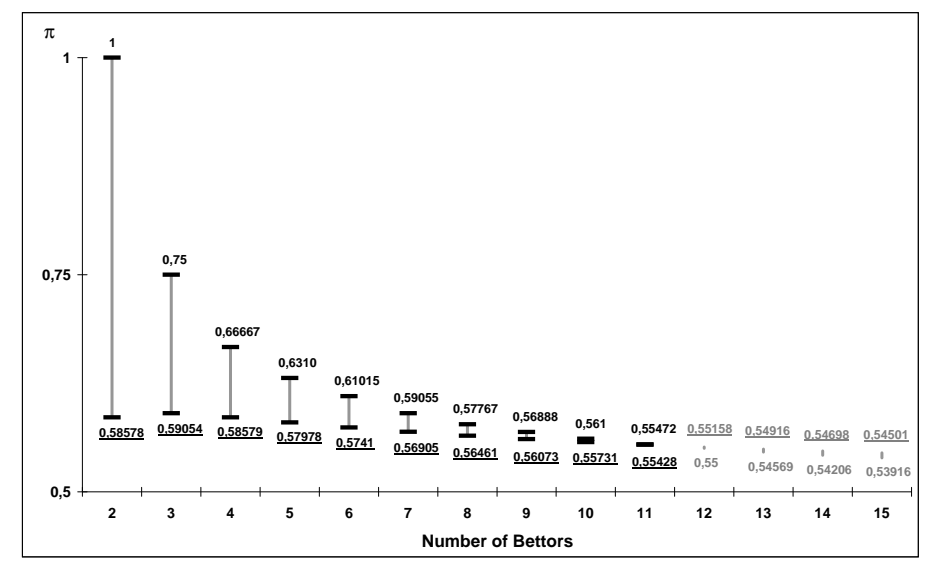

Figure 1: Intervals of Qualities of Information Allowing a Perfectly Revealing Equilibrium to Exist.

While a perfectly revealing equilibrium usually fails to exist, bettors will doubtlessly follow their own signal when the majority of previous decisions is not too pronounced. That is, the emergence of informative bets crucially relies on the observed history. A combination of uninformative bets (herd and contrarian bets) and informative bets may constitute an equilibrium. In the next subsection we characterize other equilibria than the perfectly revealing ones with two and three bettors.

\subsection{Characterization of Equilibria with Two and Three Bettors}

Proposition 8 Consider the sequential betting game with two bettors. There exists a perfectly revealing equilibrium if and only if $\pi \geq \frac{\sqrt{2}}{1+\sqrt{2}} \simeq 0.586$. Otherwise, there is an equilibrium where the first bettor always follows his signal and the second bets against the first bettor's choice whatever his signal. Those equilibria are sequential equilibria (in the sense of Kreps and Wilson, 1982), and for generic values of $\pi$ there is no other sequential equilibrium. 
In conformity with Proposition 8, the first player always bets accordingly to his signal. On the contrary, if a bettor takes his decision in the second position, then his strategy depends on the quality of the market's information. If the quality is low ( $\pi$ is low), then he always plays the current longshot (contrarian behavior). Otherwise, if the quality is good ( $\pi$ is high), then he always follows his signal. Hence, herd behavior is not possible with only two bettors. The reason is that two different signals cancel each other out and, since there is a negative payoff externality, the second bettor never follows the first bettor by betting against his own private signal. When the parimutuel betting market involves more than two players we will see that past histories can overwhelm later bettors' signals if $\pi$ is relatively high which entails the occurrence of herding behavior. The following proposition characterizes the set of all equilibria in which the first two bettors always follow their private signals.

Proposition 9 Consider the sequential betting game with three bettors. Assume that the first and the second bettors always follow their private signals, i.e., $\sigma_{1}\left(q^{H}\right)=\sigma_{2}\left(H^{\prime} ; q^{H}\right)=H$ for all $H, H^{\prime} \in\{A, B\}$.

- If $0.547 \simeq 1-2\left(\frac{2}{3(\sqrt{177}-9)}\right)^{1 / 3}+\frac{\left(\frac{1}{2}(\sqrt{177}-9)\right)^{1 / 3}}{3^{2 / 3}}<\pi<\frac{\sqrt[3]{3}}{1+\sqrt[3]{3}} \simeq 0.591$, then $\sigma$ is an equilibrium iff the third bettor uses a contrarian behavior, i.e., $\sigma_{3}\left(H, H ; q_{3}\right)=\bar{H}$ for all $q_{3} \in Q_{3}$ and $\sigma_{3}\left(H, \bar{H} ; q^{H}\right)=\sigma_{3}\left(\bar{H}, H ; q^{H}\right)=H$ for all $H \in\{A, B\}$.

- If $0.591 \simeq \frac{\sqrt[3]{3}}{1+\sqrt[3]{3}}<\pi<3 / 4$, then $\sigma$ is an equilibrium iff the third bettor always follows his signal, i.e., $\sigma$ is perfectly revealing.

- If $\pi>3 / 4$, then $\sigma$ is an equilibrium iff the third bettor herds, i.e., $\sigma_{3}\left(H, H ; q_{3}\right)=H$ for all $q_{3} \in Q_{3}$ and $\sigma_{3}\left(H, \bar{H} ; q^{H}\right)=\sigma_{3}\left(\bar{H}, H ; q^{H}\right)=H$ for all $H \in\{A, B\}$.

Contrary to the 2-bettor case, herd behavior is now possible when the quality of information is high. The behavior of the third bettor in the equilibria described by Proposition 9 is relatively intuitive. If the quality of information is low, then the negative effect of the odds against the favorite dominates the belief that this horse will win, and thus the third bettor bets on the longshot whatever his private information. If the quality of information is high, then the third bettor strongly believes that the favorite will win, and thus he bets on the favorite and also disregards his private information. For intermediate qualities of information, neither information externalities nor payoff externalities dominate, and thus the third bettor follows his signal.

There are other equilibria than those described by Proposition 9. Unfortunately, those equilibria may coincide, i.e., different types of equilibria may exist for generic qualities of information. For example, it can be shown that if $\pi \leq \frac{\sqrt{2}}{1+\sqrt{2}} \simeq 0.586$, then there is an equilibrium where the first bettor follows his signal and the other bettors adopt a contrarian behavior. For higher number of bettors, a plethora of other equilibria coexist. In the next section we consider less sophisticated decision rules to characterize unique dynamic behaviors with a larger number of bettors.

\section{Dynamic of Bets and Information Aggregation with Myopic Bettors}

In this final section we consider that the parimutuel betting market is composed of myopic players who cannot carry out game-theoretic lookahead. In other words, myopic bettors do not anticipate the signaling effect of their bets on future bets. In particular, they believe that 
odds resulting from their bets are the final odds. Nonetheless, while myopic bettors are not endowed with forward looking behaviors, they still perform backward looking behaviors. That is, myopic bettors maximize a particular perceived payoff and they update their information via Bayes' rule, knowing that all previous bettors are myopic bettors.

Considering myopic bettors has at least three advantages for the analysis of parimutuel betting markets. First, equilibrium outcomes are unique, indifference cases notwithstanding. Second, we are able to provide an exhaustive analysis of bettors' behavior in markets with a significant number of bettors. Finally, direct comparisons with previous studies on herd behavior in financial markets where no lookahead behavior is required can be made (see Section 6).

While optimal strategies are easier to compute with myopic bettors, we will see that conditions for the existence of a perfectly revealing equilibrium are similar to those with fully rational bettors. Additionally, herd and contrarian behaviors also arise, so the most important and interesting phenomena obtained before are not lost here.

The new sequential game with myopic bettors is modeled as follows. Given a sequence of bets up to player $i, s^{i} \in S^{i}$, the odds of period $i$ against horse $H \in\{A, B\}$ is

$$
O_{H}\left(s^{i}\right)=\frac{i-h\left(s^{i}\right)}{h\left(s^{i}\right)}=\frac{\bar{h}\left(s^{i}\right)}{h\left(s^{i}\right)} .
$$

For all $H \in\{A, B\}$, the (perceived) payoff of each myopic bettor $i \in N$ is

$$
u_{i}\left(s^{i}, \theta\right)= \begin{cases}O_{H}\left(s^{i}\right)+1=\frac{i}{h\left(s^{i}\right)} & \text { if } s_{i}=H \text { and } \theta=\theta_{H} \\ 0 & \text { if } s_{i}=H \text { and } \theta \neq \theta_{H}\end{cases}
$$

So defined, myopic bettors incorporate the direct effect of their choices on the current betting odds, but not on later odds movements. ${ }^{8}$ The information structure and beliefs are defined exactly as in the previous section but the (perceived) expected utility takes a quite simpler form because current bettors do not forecast future bettors' behavior:

$$
U_{i}\left(H \mid s^{i-1} ; q_{i}\right)=\mu_{i}\left(\theta_{H} \mid s^{i-1} ; q_{i}\right) \frac{i}{1+h\left(s^{i-1}\right)},
$$

where, as before, $\mu_{i}\left(\theta \mid s^{i-1}, q_{i}\right)$ is given by Equation (6). ${ }^{9}$ For all $H \in\{A, B\}$, we obtain $U_{i}\left(H \mid s^{i-1} ; q_{i}\right)>U_{i}\left(\bar{H} \mid s^{i-1} ; q_{i}\right)$ iff

$$
\mu_{i}\left(\theta_{H} \mid s^{i-1} ; q_{i}\right)>\frac{h\left(s^{i-1}\right)+1}{i+1}
$$

which corresponds to Lemma 4 with $i=n$.

Since rewards decrease with the number of individuals having chosen the correct option, it is easy to see that long-run herding is impossible in parimutuel betting markets. Indeed, if long-run herding would occur in some period $k$, then all bettors after some history $s^{k} \in$ $S^{k}$ would bet on some horse $H$ independently of their private signal. In that case, beliefs would remain constant after period $k$, whereas betting odds against horse $H \in\{A, B\}$ would continuously decline. Therefore, there is unavoidably a period after period $k$ in which a bettor switches by betting on the other horse, a contradiction with the fact that they all follow the

\footnotetext{
${ }^{8}$ We can easily remark that if myopic bettors were playing the simultaneous move game, then, whatever the parameters of the market, they would all follow their private signal, resulting in the fully informative equilibrium obtained in Section 3.

${ }^{9}$ Of course, outside equilibrium beliefs are irrelevant for the analysis of equilibrium outcomes with myopic bettors.
} 
crowd. Nevertheless, as informational externalities coexist with negative payoff externalities, the emergence of pronounced herd behavior cannot be prevented.

As in the previous section, we investigate in the following subsection conditions for the existence of a perfectly revealing equilibrium. Similar conditions are found; in particular, when the number of bettors increase, the perfectly revealing equilibrium disappears.

\subsection{Perfectly Revealing Equilibrium}

Assume that bettors before $i$ followed their private signal and that bettor $i$ receives the signal $q^{H}$ for some $H \in\{A, B\}$. Then, using Lemma 5 on page 9 , Inequality (10) becomes equivalent to $\frac{\pi^{2 h+2-i}}{\pi^{2 h+2-i}+(1-\pi)^{2 h+2-i}}>\frac{h+1}{i+1}$, where $h=h\left(s^{i-1}\right)$. This inequality can be rewritten as

$$
\begin{array}{ll}
\pi>\frac{(h+1)^{\frac{1}{2 h+2-i}}}{(h+1)^{\frac{1}{2 h+2-i}}+(i-h)^{\frac{1}{2 h+2-i}}} & \text { if } h>\frac{i-2}{2} \\
\pi<\frac{(h+1)^{\frac{1}{2 h+2-i}}}{(h+1)^{\frac{1}{2 h+2-i}}+(i-h)^{\frac{1}{2 h+2-i}}} & \text { if } h<\frac{i-2}{2} .
\end{array}
$$

It is easy to show that the first bettor always follows his signal. The second bettor always follows his signal iff $\pi \geq \frac{\sqrt{2}}{1+\sqrt{2}}$. The third bettor always follows his signal iff $\pi \geq \frac{\sqrt[3]{3}}{1+\sqrt[3]{3}}$ $\left(>\frac{\sqrt{2}}{1+\sqrt{2}}\right)$ and $\pi \leq 3 / 4$. Hence, with two or three bettors the conditions for a perfectly revealing equilibrium to exist are the same as with fully rational bettors. Actually, by computing numerically the conditions for bettors $i=2$ to 15 to follow their private information whatever the observed history, we find slightly stronger conditions than those represented in Figure 1. More precisely, an equilibrium in which the first $i$ bettors follow their signal exists iff $\pi$ belongs to the intervals $[\underline{\pi}(j), \bar{\pi}(j)]$ represented in Figure 1 for all $j \leq i$. Hence, when the number of bettors exceeds 7 , no perfectly revealing equilibrium exists. The intuition is that the strongest conditions are imposed on the last bettor and, at the perfectly revealing equilibrium, the last bettor is exactly in the same situation when all bettors are myopic as when all bettors are fully rational. In the following lines we turn to the analysis of other equilibrium outcomes in which bets are not perfectly revealing.

\subsection{Beliefs and Optimal Strategies of Myopic Bettors}

Without necessarily assuming perfectly revealing strategies, the form of bettor $i$ 's posterior probability that horse $H$ wins after inferring, from the history $s^{i-1}, k_{H}\left(s^{i-1}\right)=k_{H}$ signals favoring horse $H\left(q^{H}\right.$ signals) and $k_{\bar{H}}\left(s^{i-1}\right)=k_{\bar{H}}$ signals favoring horse $\bar{H}$ ( $q^{\bar{H}}$ signals) is

$$
\mu_{i}\left(\theta_{H} \mid s^{i-1} ; q_{i}\right)= \begin{cases}\frac{\pi^{k} H^{+1}(1-\pi)^{k} \bar{H}}{\pi^{k} H^{+1}(1-\pi)^{k} \bar{H}+(1-\pi)^{k} H^{+1} \pi^{k} \bar{H}} & \text { if } q_{i}=q^{H} \\ \frac{\pi^{k} H(1-\pi)^{k} \bar{H}^{+1}}{\pi^{k_{H}}(1-\pi)^{k^{+}}{ }^{+1}+(1-\pi)^{k} H \pi^{k} \bar{H}^{+1}} & \text { if } q_{i}=q^{\bar{H}}\end{cases}
$$

where we necessarily have $k_{H}+k_{\bar{H}} \leq i-1$. Of course, $\mu_{i}\left(\theta_{H} \mid s^{i-1} ; q_{i}\right)$ is increasing with the difference $k_{H}-k_{\bar{H}}$, and $k_{H}=k_{\bar{H}}$ implies $\mu_{i}\left(\theta_{H} \mid s^{i-1} ; q_{i}\right)=1 / 2$. The procedure we used to compute the optimal strategy of each bettor depending on his signal and the history he has observed is the following.

Bettor 1. Let $k_{H}(\emptyset)=k_{\bar{H}}(\emptyset)=0$. For all $q_{1} \in Q_{1}$ and $H \in\{A, B\}$, we obtain $\mu_{1}\left(\theta_{H} \mid q_{1}\right)$ from Equation (11) and $\sigma_{1}\left(q_{1}\right)$ from the best response deduced from Inequality (10). Of course, we get here $\mu_{1}\left(\theta_{H} \mid q^{H}\right)=\pi, \mu_{1}\left(\theta_{H} \mid q^{\bar{H}}\right)=1-\pi$, and $\sigma_{1}\left(q^{H}\right)=H$ for all $H \in\{A, B\}$. 
Bettor $i$. For all $s^{i-1} \in S^{i-1}$ and $H \in\{A, B\}$, let

$$
k_{H}\left(s^{i-1}\right)= \begin{cases}k_{H}\left(s^{i-2}\right)+1 & \text { if } \sigma_{i-1}\left(s^{i-2} ; q^{H}\right)=s_{i-1} \text { and } \sigma_{i-1}\left(s^{i-2} ; q^{\bar{H}}\right) \neq s_{i-1} \\ k_{H}\left(s^{i-2}\right) & \text { otherwise. }\end{cases}
$$

For all $s^{i-1} \in S^{i-1}, q_{i} \in Q_{i}$ and $H \in\{A, B\}$, we obtain $\mu_{i}\left(\theta_{H} \mid s^{i-1} ; q_{i}\right)$ from Equation (11) and $\sigma_{i}\left(s^{i-1} ; q_{i}\right)$ from the best response deduced from Inequality (10).

Computing this procedure for given qualities of information $\pi \in] 0.5,1[$ allows us to obtain beliefs and optimal strategies of each bettor in the sequence for all histories and all profiles of signals. ${ }^{10}$ We have represented the set of all possible outcomes for the first five bettors in Figures 2, 3, and 4, where in the first column " $a$ " (" $b$ ", resp.) in position $i \in\{1, \ldots, 5\}$ corresponds to a signal $q^{A}\left(q^{B}\right.$, resp.) for bettor $i .{ }^{11}$ As in the previous section, we see that there is a preponderance of uninformative bets with low qualities of information due to contrarian behaviors and with high qualities of information due to herd behavior. All bets are always informative only for qualities of information between approximately 0.59 and 0.63. Hence, at least in the short-run, contrarian and herd behaviors arise whether or not bettors are endowed with forward looking behavior. In the next subsection we show that herd behavior leads to disproportionate mispricing up to at least 15 periods in identifiable states of the world.

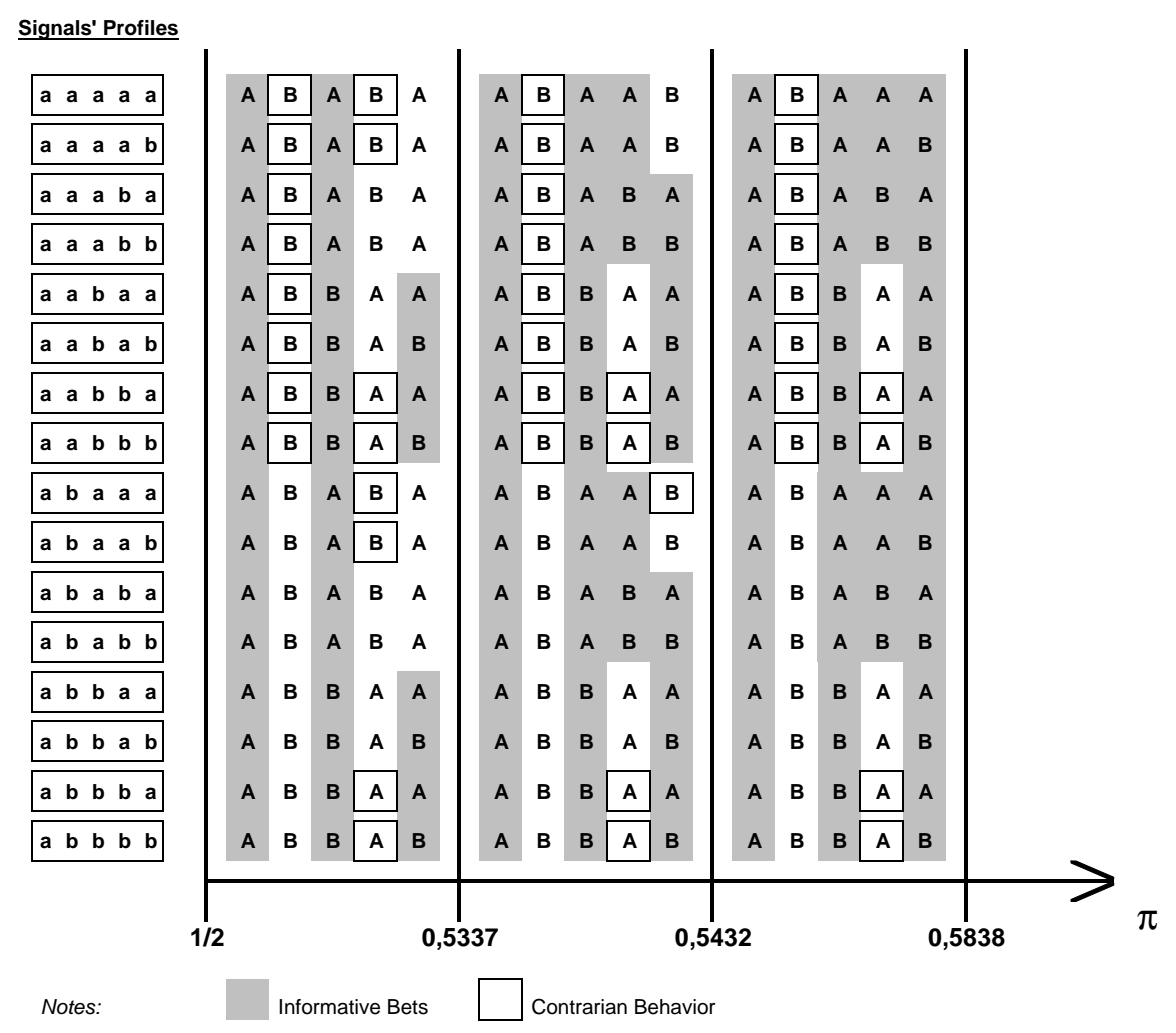

Figure 2: Set of Outcomes for the First Five Myopic Bettors with Low Values of $\pi$.

\footnotetext{
${ }^{10}$ The computational program is available from the authors upon request.

${ }^{11}$ When a bettor is indifferent between betting on horse $A$ and betting on horse $B$ we assume as a tie-breaking rule that he bets on horse $B$.
} 


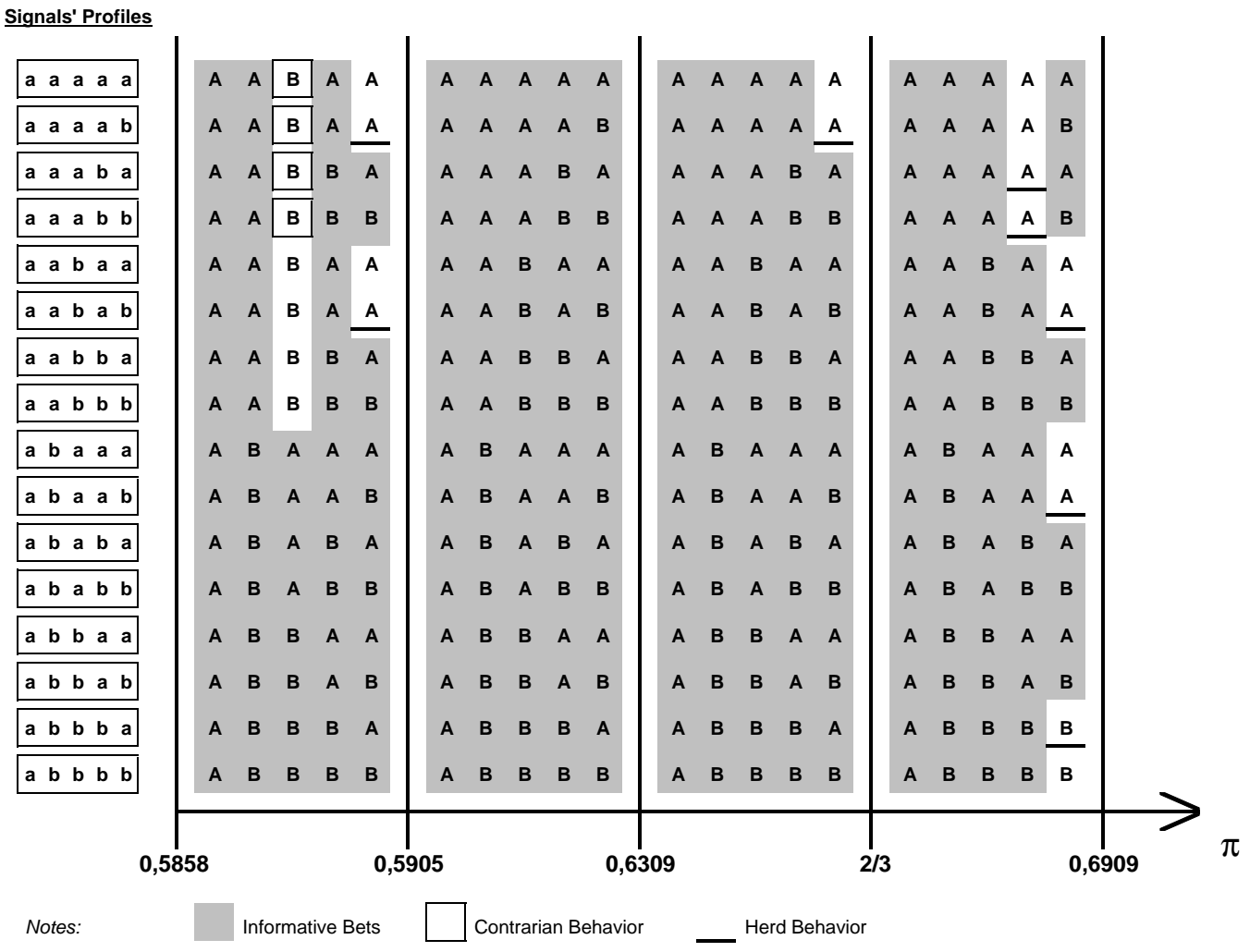

Figure 3: Set of Outcomes for the First Five Myopic Bettors with Middle Values of $\pi$.

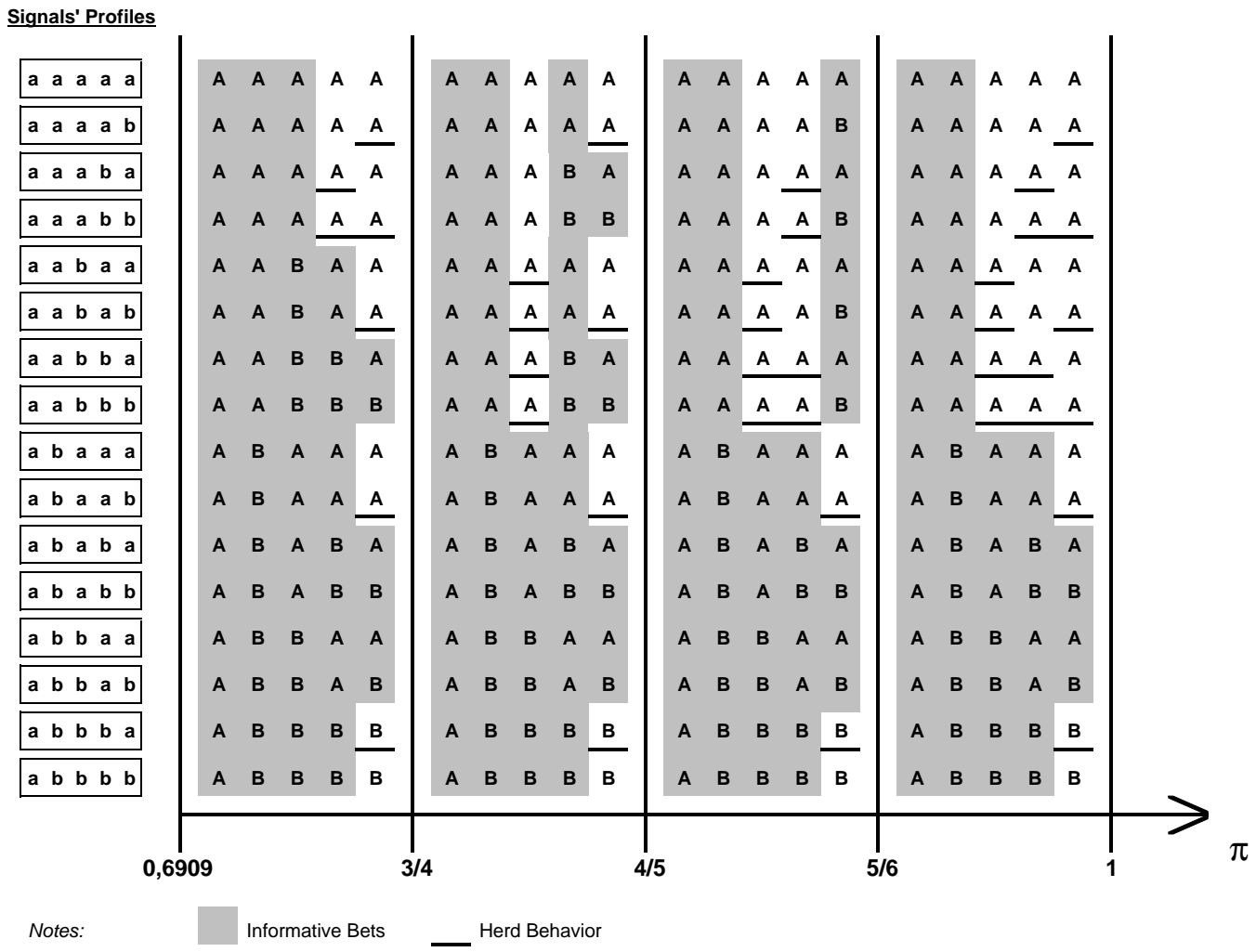

Figure 4: Set of Outcomes for the First Five Myopic Bettors with High Values of $\pi$. 


\subsection{Some Examples of Informational Inefficiencies}

Since short-run herding and non-informative bets in general can arise, valuable information may be lost in the parimutuel betting market. In this respect, it seems worthwhile to investigate more deeply whether significant bandwagon effects in the betting odds can be created. More generally, we now examine if herd and contrarian behaviors (which are non-informative behaviors by definition) can lead to significant mispricing. To address this issue, we compare implicit prices set in the market - represented by horses' subjective probabilities - with objective probabilities.

The subjective probability of period $i$ that horse $H$ wins the race depends on the betting odds established in period $i$, and is given by

$$
P_{H}^{S}\left(s^{i}\right) \equiv \frac{h\left(s^{i}\right)}{i}=\frac{1}{O_{H}\left(s^{i}\right)+1} .
$$

This variable can be viewed as the price of obtaining a claim to one unit of money in the event that horse $H$ wins the race, and thus it is also called the (equilibrium) implicit price for horse $H$ in period $i$ if $s^{i}$ is an equilibrium history of bets. To simplify the notations, let $P_{H}^{S}\left(s\left(\sigma \mid q^{i}\right)\right)=P_{H}^{S}\left(q^{i}\right)$, where $\sigma$ is an equilibrium strategy profile. The objective probability of period $i$ that horse $H$ wins the race is given by

$$
P_{H}^{O}\left(q^{i}\right) \equiv \operatorname{Pr}\left(\theta_{H} \mid q^{i}\right),
$$

where $q^{i}=\left(q_{1}, \ldots, q_{i}\right)$ is the vector of signals received by all bettors up to period $i$. Hence, $P_{H}^{O}\left(q^{i}\right)$ is the belief about the winning chance of horse $H$ for an agent who would be able to observe the signals of all bettors up to period $i$. Said differently, if bettors before bettor $i$ use the perfectly revealing strategy, then $\mu_{i}\left(\theta_{H} \mid s^{i-1} ; q_{i}\right)=P_{H}^{O}\left(q^{i}\right)$.

By means of simulations, we now illustrate how inefficient can win pool shares (implicit prices) be in forecasting outcomes in a sequential parimutuel betting market. The initial conditions for the first simulation are the following: the quality of information is $\pi=0.85$ and the profile of private signals is $q^{15}=\left(q^{B}, q^{B}, q^{A}, q^{A}, q^{A}, q^{B}, q^{A}, q^{A}, q^{A}, q^{A}, q^{A}, q^{A}, q^{A}, q^{A}, q^{A}\right)$. Figure 5 on the following page shows the evolution of bettors' beliefs about horse $A, \mu_{i}\left(\theta_{A} \mid\right.$

$\left.s^{i-1} ; q_{i}\right)$, the evolution of the implicit price for horse $A, P_{A}^{S}\left(s^{i}\right)$, and the evolution of the objective probability of horse $A, P_{A}^{O}\left(q^{i}\right)$, up to period 15. After fifteen periods of bets, the implicit price for horse $A$ and bettors' beliefs about the winning chance of horse $A$ are close to zero, whereas the objective probability of horse $A$ is close to 1 . Here, a sufficiently excess of bets on horse $B$ at the beginning of the sequence leads bettors to believe that horse $B$ is more likely to win, regardless of their own private information. Hence, informational efficiency of betting odds is severely disrupted due to long sequences of imitative bets.

The quality of information used in the first simulation is relatively extreme and one might believe that such inefficiencies cannot arise with intermediate qualities of information. However, this is not the case. Figure 6 on the next page shows the 15-period moving of beliefs, implicit prices and objective probabilities of horse $A$ with the sequence of private signals $q^{15}=\left(q^{A}, q^{A}, q^{A}, q^{B}, q^{B}, q^{A}, q^{B}, q^{B}, q^{B}, q^{B}, q^{B}, q^{B}, q^{A}, q^{B}, q^{B}\right)$ and with the quality of information $\pi=0.7$. Here again, significant mispricing and discrepancies between beliefs and objective probabilities are observed. Heavy bets on a horse trigger more bets on that horse, even though this horse turned out not to be the one that will win the race according to the information which is distributed among all bettors. Nevertheless, this inefficiency is stronger to obtain with $\pi=0.7$ than with $\pi=0.85$ since three initial decisions on the "wrong" horse are necessary to generate herding. For example, as illustrated in Figure 7 on page 18, prices set in the market can converge to the fundamentals for other profiles of signals. Of course, 


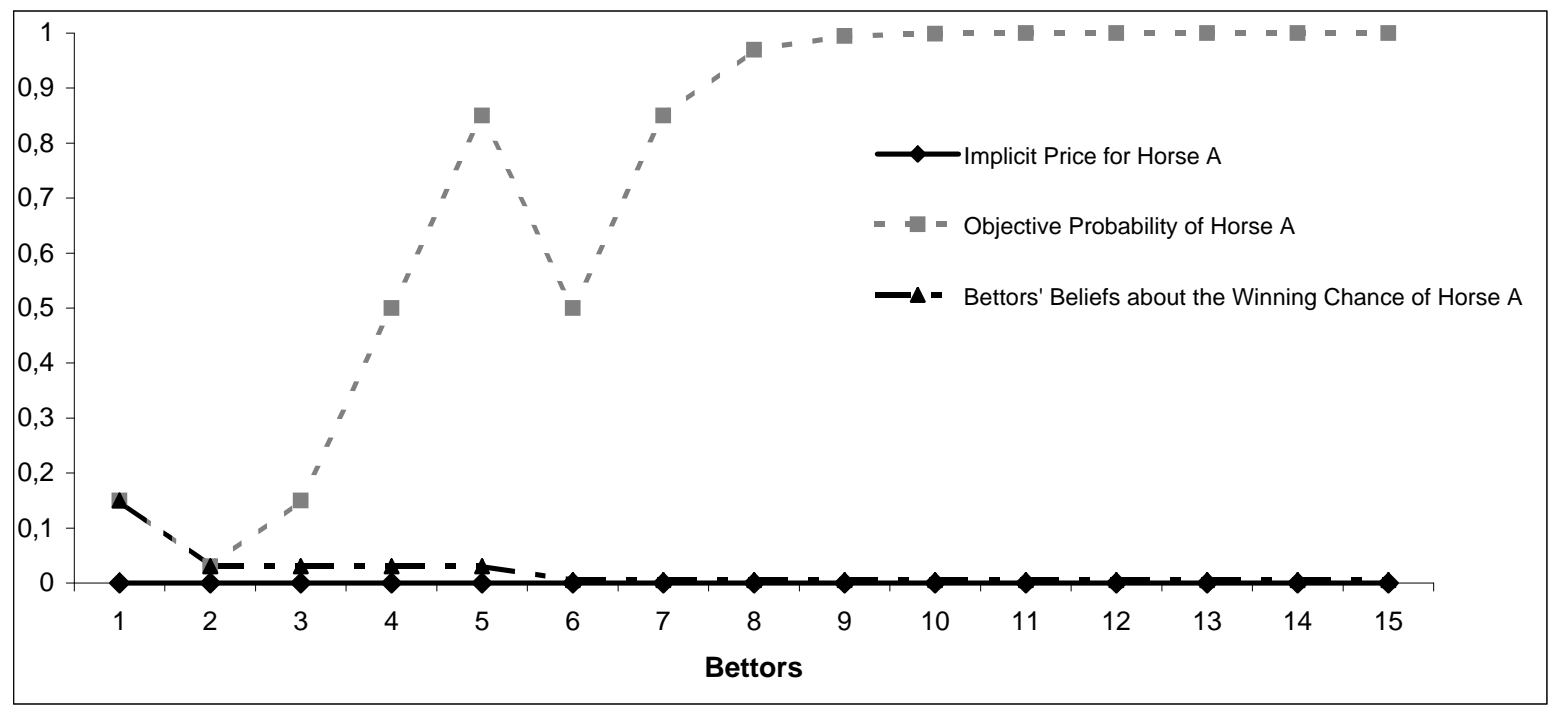

Figure 5: Evolution of Beliefs, Implicit Prices, and Objective Probabilities of Horse $A$ up to Bettor 15 with $\pi=0.85$ and $q^{15}=\left(q^{B}, q^{B}, q^{A}, q^{A}, q^{A}, q^{B}, q^{A}, q^{A}, q^{A}, q^{A}, q^{A}, q^{A}, q^{A}, q^{A}, q^{A}\right)$.

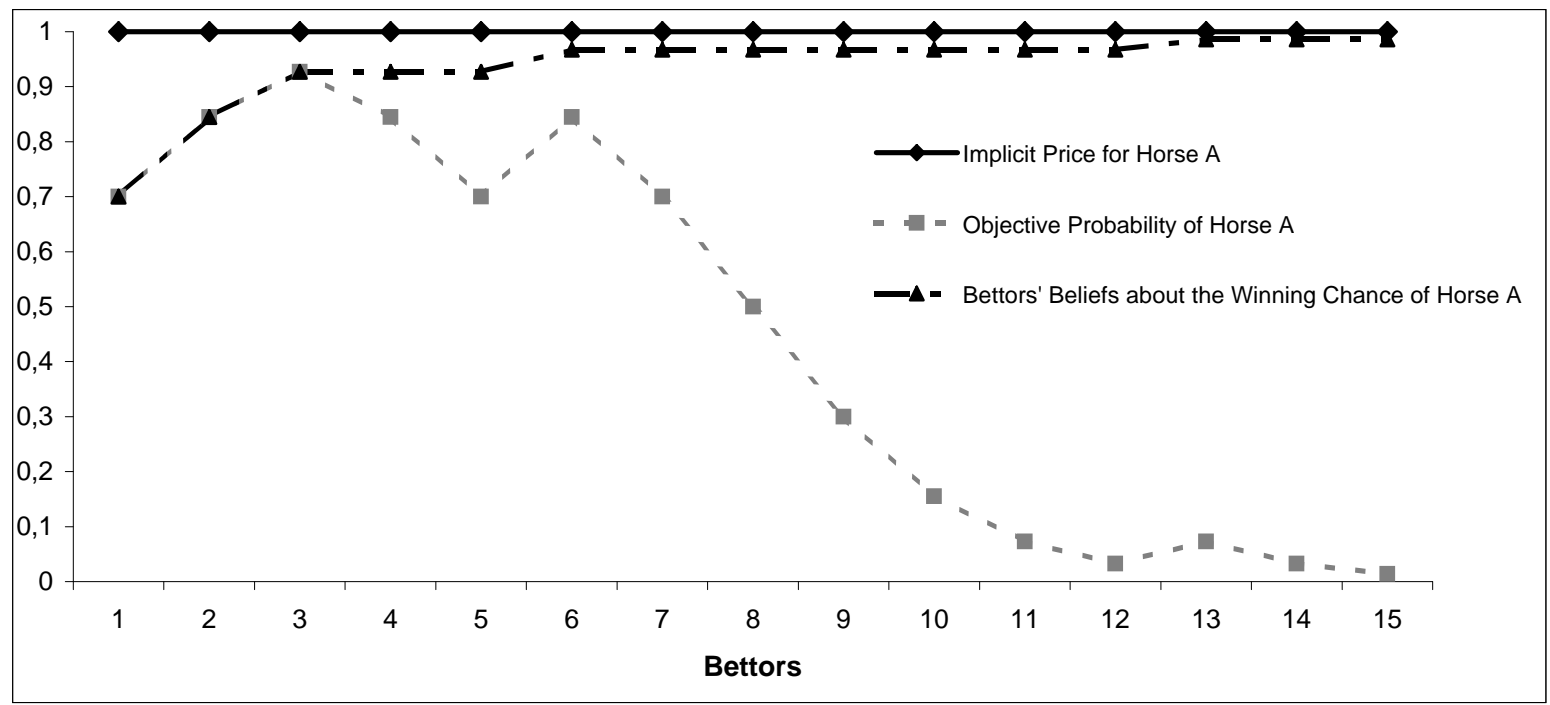

Figure 6: Evolution of Beliefs, Implicit Prices, and Objective Probabilities of Horse $A$ up to Bettor 15 with $\pi=0.70$ and $q^{15}=\left(q^{A}, q^{A}, q^{A}, q^{B}, q^{B}, q^{A}, q^{B}, q^{B}, q^{B}, q^{B}, q^{B}, q^{B}, q^{A}, q^{B}, q^{B}\right)$.

implicit prices adjust relatively slowly after some periods because of the relative rigidity of betting odds.

Finally, it is worth noticing that significant mispricing is possible even if the first decisions correctly reflect the fundamentals. Indeed, as illustrated in Figure 8 on the next page, herding might arise later due to non-informative bets during the last five periods. In the next subsection we examine the relevance of the above examples on the average efficiency of the market in aggregating bettors' private information. 


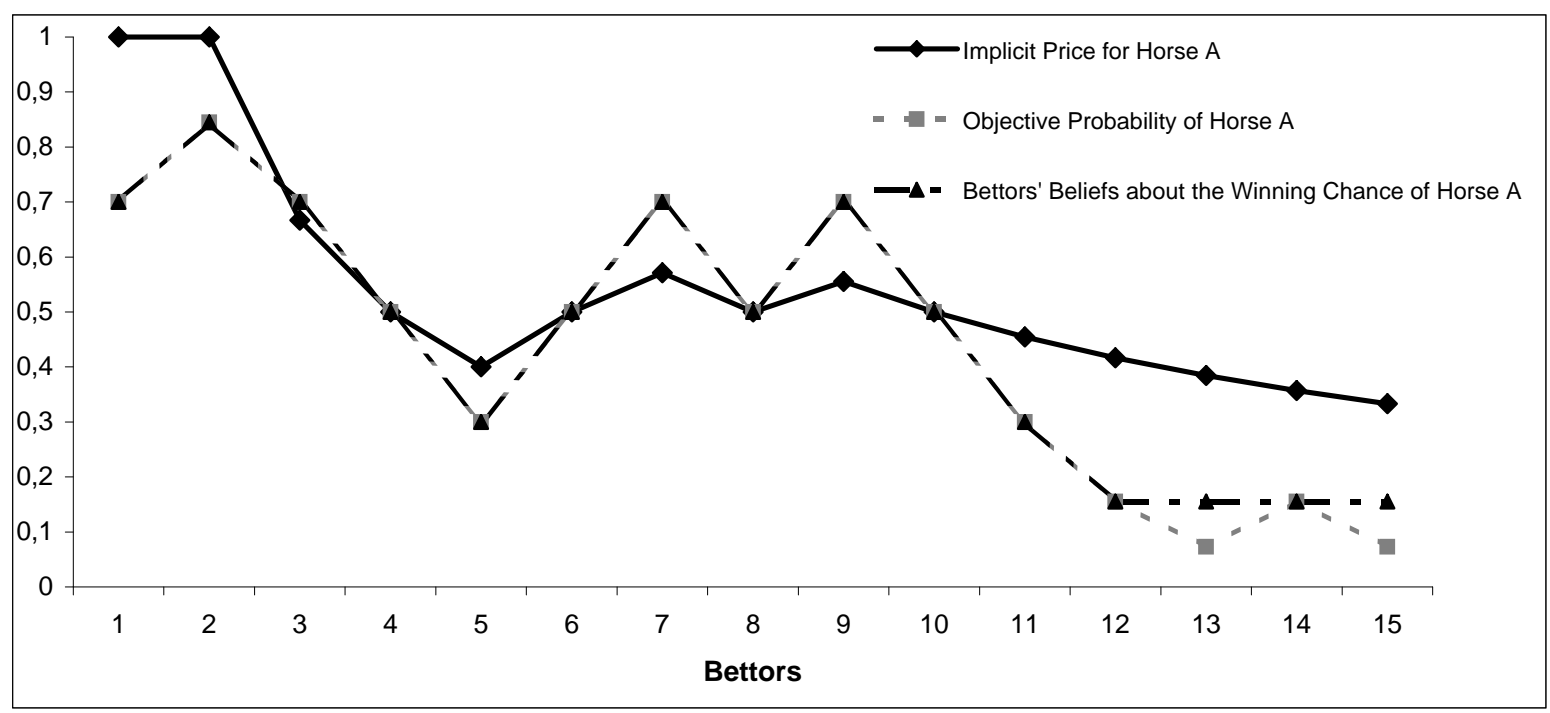

Figure 7: Evolution of Beliefs, Implicit Prices, and Objective Probabilities of Horse $A$ up to Bettor 15 with $\pi=0.70$ and $q^{15}=\left(q^{A}, q^{A}, q^{B}, q^{B}, q^{B}, q^{A}, q^{A}, q^{B}, q^{A}, q^{B}, q^{B}, q^{B}, q^{B}, q^{A}, q^{B}\right)$.

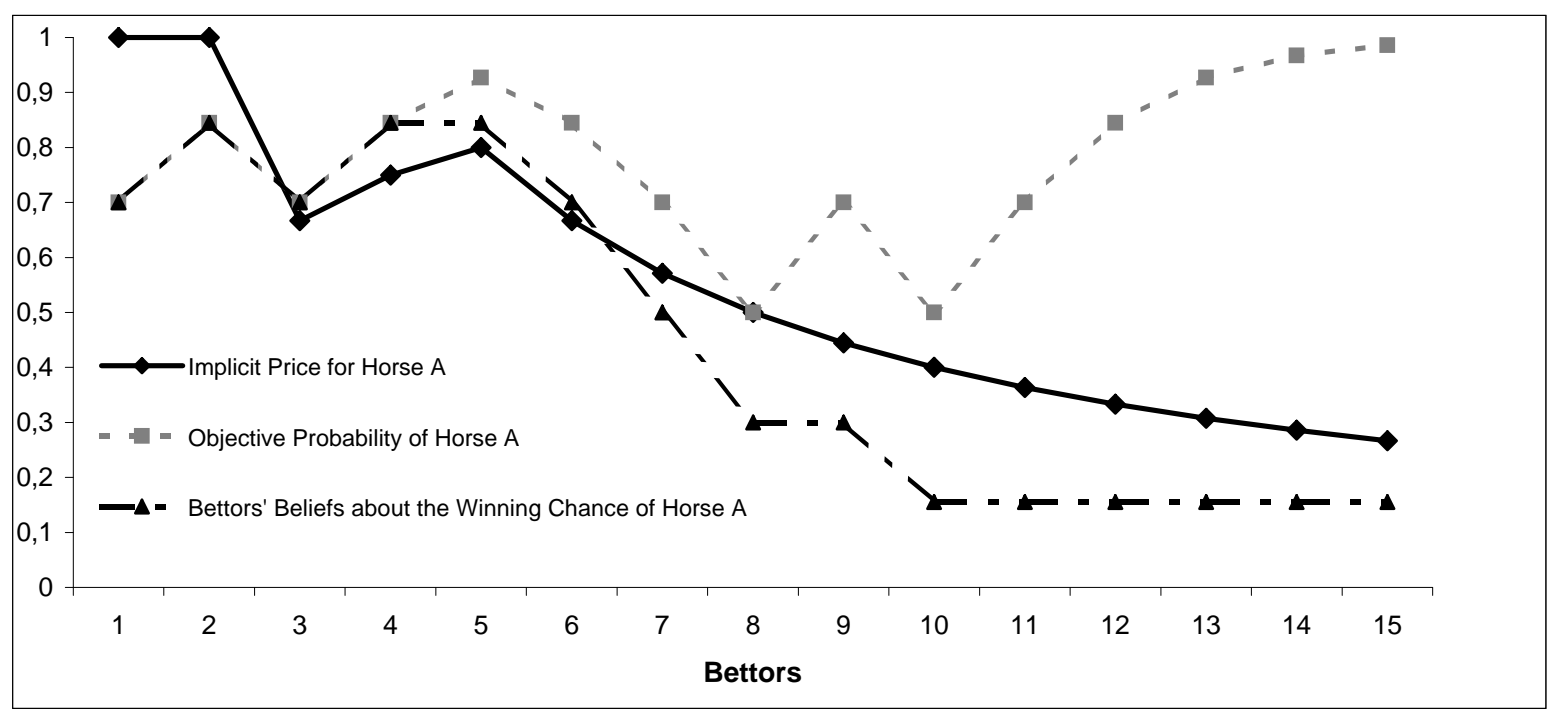

Figure 8: Evolution of Beliefs, Implicit Prices, and Objective Probabilities of Horse $A$ up to Bettor 15 with $\pi=0.70$ and $q^{15}=\left(q^{A}, q^{A}, q^{B}, q^{A}, q^{A}, q^{B}, q^{B}, q^{B}, q^{A}, q^{B}, q^{A}, q^{A}, q^{A}, q^{A}, q^{A}\right)$. 


\subsection{Measures of Information Aggregation in Prices}

According to the previous examples, betting odds aggregate only partially the information privately held by market participants in some states of the world. This informational inefficiency leads to high discrepancies between fundamental prices (objective probabilities) and equilibrium prices (subjective probabilities). We now present general measurements of information aggregation. In this respect, we first describe the two backgrounds against which information aggregation measurements will be made. Then we explain the measurement method we have adopted to compare the levels of information in different patterns of prices. As our analyses are restricted to parimutuel betting markets which involve at most fifteen players, we finally discuss the extent to which these results apply to large betting markets.

Our first baseline is an artificial parimutuel betting market where each bettor has access to all the private information of previous bettors (including his own private information). The dynamic of prices in such a "full communication" market is relatively simple to characterize. Indeed, each bettor $i$ bets on horse $H$ if $\operatorname{Pr}\left(\theta_{H} \mid q^{i}\right)>\frac{h\left(s^{i-1}\right)+1}{i+1}$. When the number of bettors increases, the implicit price for horse $H$ tends to the objective probability which, according to the law of large numbers, tends to 1 if the winning horse is horse $H$, and tends to 0 otherwise. The final full communication implicit price for horse $H \in\{A, B\}$ is denoted by $P_{H}^{F C}\left(q^{n}\right)$.

As an alternative baseline we consider a parimutuel betting market where individuals make decisions only on their own private information. In other words, players do not infer any information from past bets, they simply respond optimally to the current odds given their private signal. Formally, each bettor $i$ bets on horse $H$ if $\operatorname{Pr}\left(\theta_{H} \mid q_{i}\right)>\frac{h\left(s^{i-1}\right)+1}{i+1}$. We denote by $P_{H}^{P I}\left(q^{n}\right)$ the final private information implicit price for horse $H \in\{A, B\}$. Though some type of merging of information will be reflected in the private information price, there is no explicit information aggregation as part of the behavior of the market.

Since measures of the degree of aggregation involve comparisons of distributions, we adopt the measure proposed by Würtz (1997) to determine when one price pattern is close in information content to another price pattern. ${ }^{12}$ More precisely, we study the evolution of the average distances

$$
\begin{aligned}
V^{1}(\pi, n) & =\sum_{q^{n} \in Q^{n}} \operatorname{Pr}\left(q^{n}\right)\left|P_{H}^{F C}\left(q^{n}\right)-P_{H}^{S}\left(q^{n}\right)\right|, \\
V^{2}(\pi, n) & =\sum_{q^{n} \in Q^{n}} \operatorname{Pr}\left(q^{n}\right)\left|P_{H}^{F C}\left(q^{n}\right)-P_{H}^{P I}\left(q^{n}\right)\right|,
\end{aligned}
$$

for various values of $\pi$ and $n$. If the parimutuel betting market with myopic players is (strongform) informationally efficient, then the final equilibrium price is always equal to the final full communication price, and thus $V^{1}(\pi, n)=0$ for all $\left.\pi \in\right] 0.5,1[$ and $n>1$. On the contrary, informational inefficiency is characterized by how close are the two average distances $V^{1}(\pi, n)$ and $V^{2}(\pi, n)$ given a pair $(\pi, n)$.

We illustrate the results of our simulations by means of three examples. As such an analysis is highly time consuming we have considered at most fifteen players, and $\pi$ starts at 0.505 and ends at 0.995 by an increment of $0.05 .^{13}$ Figure 9 on the following page shows the evolution of the two distances for $\pi=0.53$. As already mentioned, low values of the quality of information induce contrarian behavior. Due to this preponderance of uninformative bets the two average distances are close to each other when the market involves less than ten players. However, the

\footnotetext{
${ }^{12}$ Würtz's (1997) function simply measures the distance between two densities. If two discrete distributions are described by their probability density functions $\left\{x_{i}\right\}_{i=1, \ldots, m}$ and $\left\{y_{i}\right\}_{i=1, \ldots, m}$ respectively, then the measure is given by $0.5 \sum_{i=1}^{m}\left|x_{i}-y_{i}\right|$.

${ }^{13}$ The simulation program is available from the authors upon request.
} 
parimutuel betting market becomes more informationally efficient as its size increases. This is not surprising as informative bets do accumulate. The same qualitative results are obtained for $\pi \in[0.505,0.59[$.

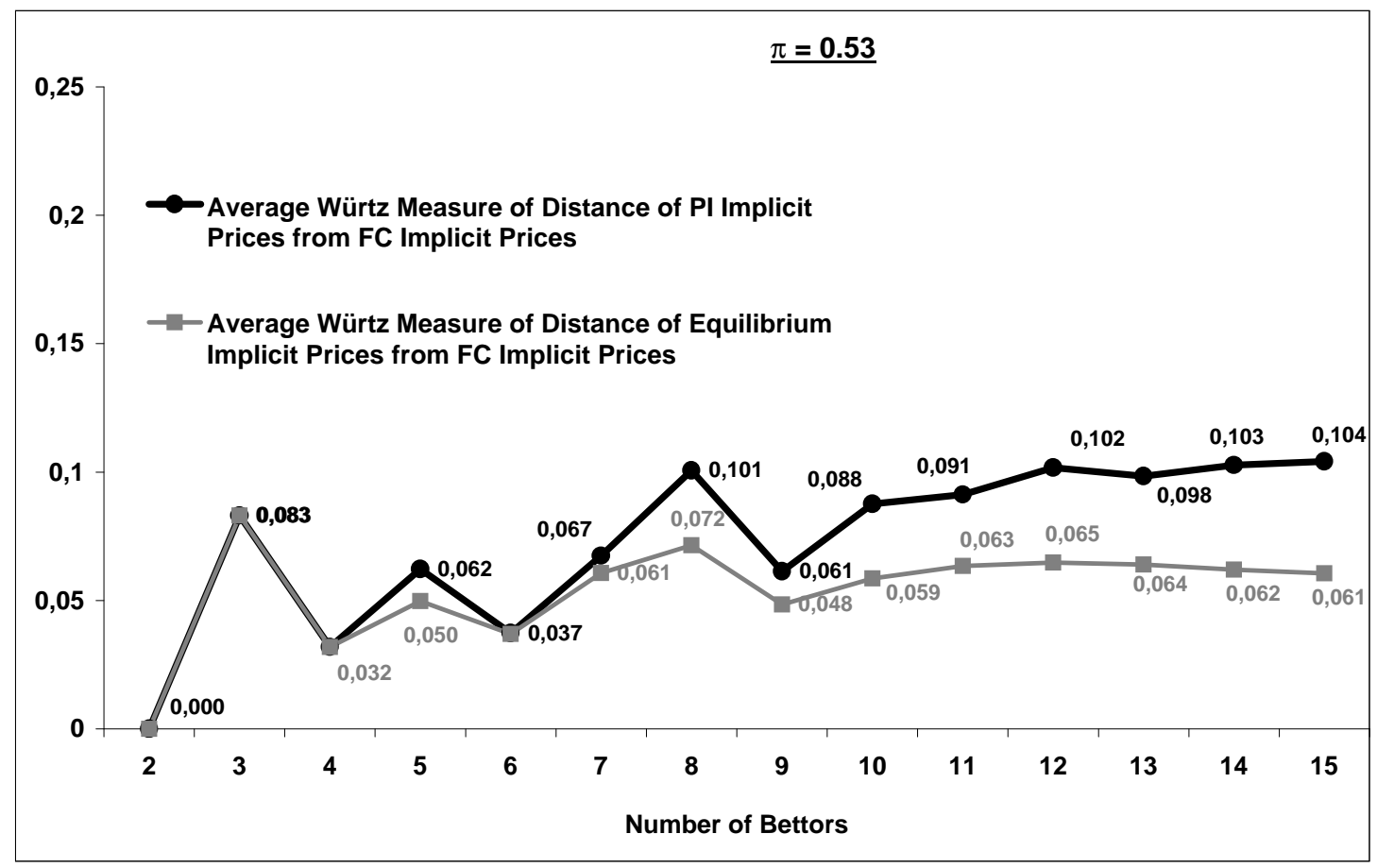

Figure 9: Evolution of Average Distances $V^{1}(\pi, n)$ and $V^{2}(\pi, n)$ up to Bettor 15 with $\pi=0.53$.

On the contrary, the parimutuel betting market seems to become less informationally efficient as its size increases for intermediate values of $\pi$. Figure 10 on the next page shows the evolution of the two distances for $\pi=0.7$ (the same qualitative results are obtained for $\pi \in[0.59,0.85[)$. As shown in the previous subsection, such a value of the quality of information entails herd behavior. Even though the degree of informational inefficiency is quite low, the preponderance of uninformative bets due to herd behavior still persists for middle-sized parimutuel betting markets.

Figure 11 on the following page shows the evolution of the two distances for $\pi=0.875$. Such a high degree of informational efficiency is typical of large values of $\pi$ as one signal already leads to a good forecast of the parimutuel betting market's outcome.

Though the parimutuel betting markets we considered in this subsection are quite small, we believe that our results extend to larger markets. Indeed, more than the magnitude of the average distance $V^{1}(\pi, n)$ and its comparison with $V^{2}(\pi, n)$, it is the dynamic of $V^{1}(\pi, n)$ which helps predicting the capacity of large parimutuel mechanisms to aggregate the information held privately by the players. In this respect, as far as extreme values of the quality of information are considered, there is a clear evidence that most of the information held by the players becomes aggregated by the dynamics of the price process. On the contrary, for intermediate values of $\pi$ the parimutuel betting market seems to do a less effective job of aggregation. The analyses conducted in this subsection have established this result at an average level and part of the examples presented in subsection 5.3 have shown that mispricing effects consistently occur under certain identifiable, but unlikely, conditions. 


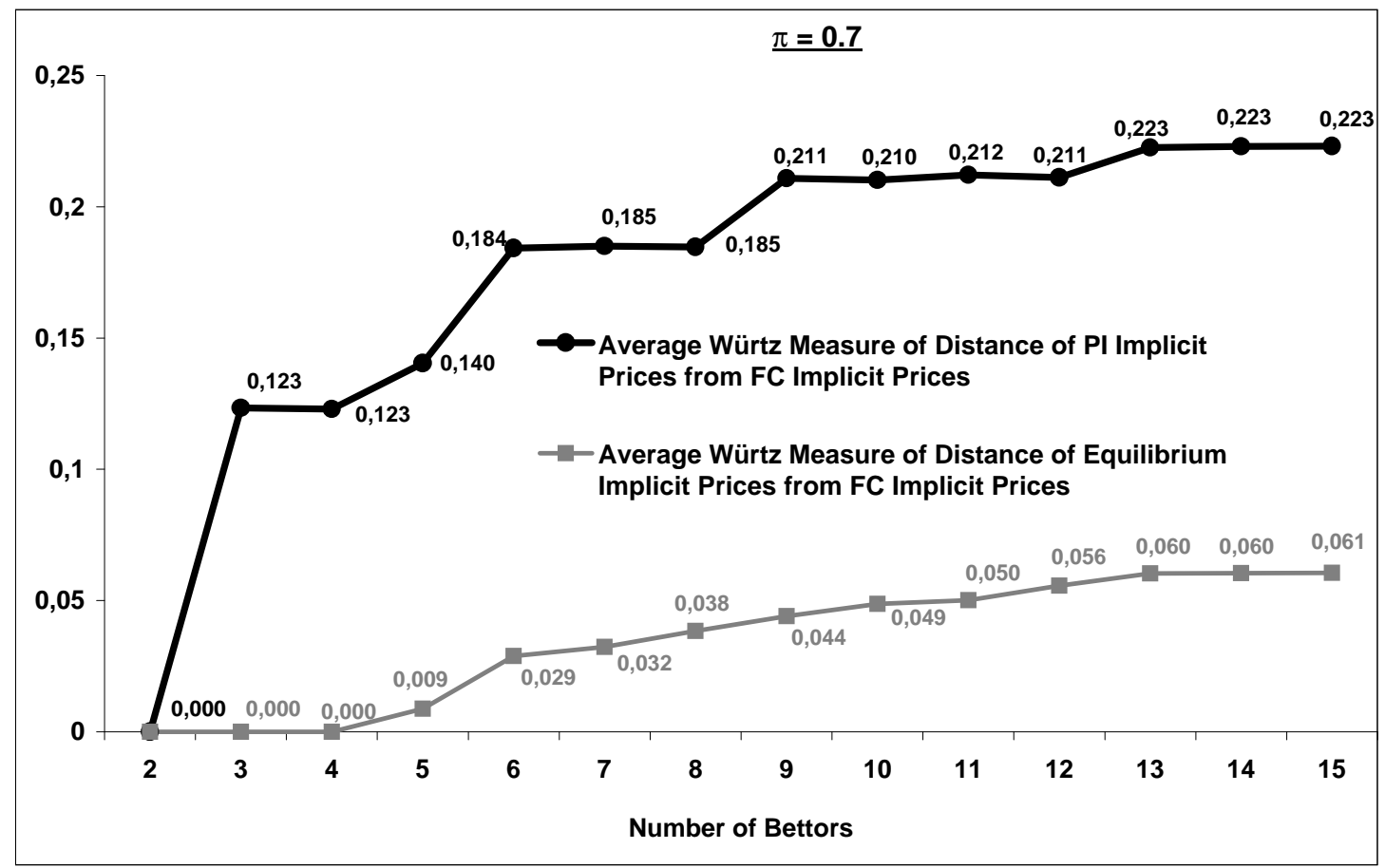

Figure 10: Evolution of Average Distances $V^{1}(\pi, n)$ and $V^{2}(\pi, n)$ up to Bettor 15 with $\pi=0.7$.

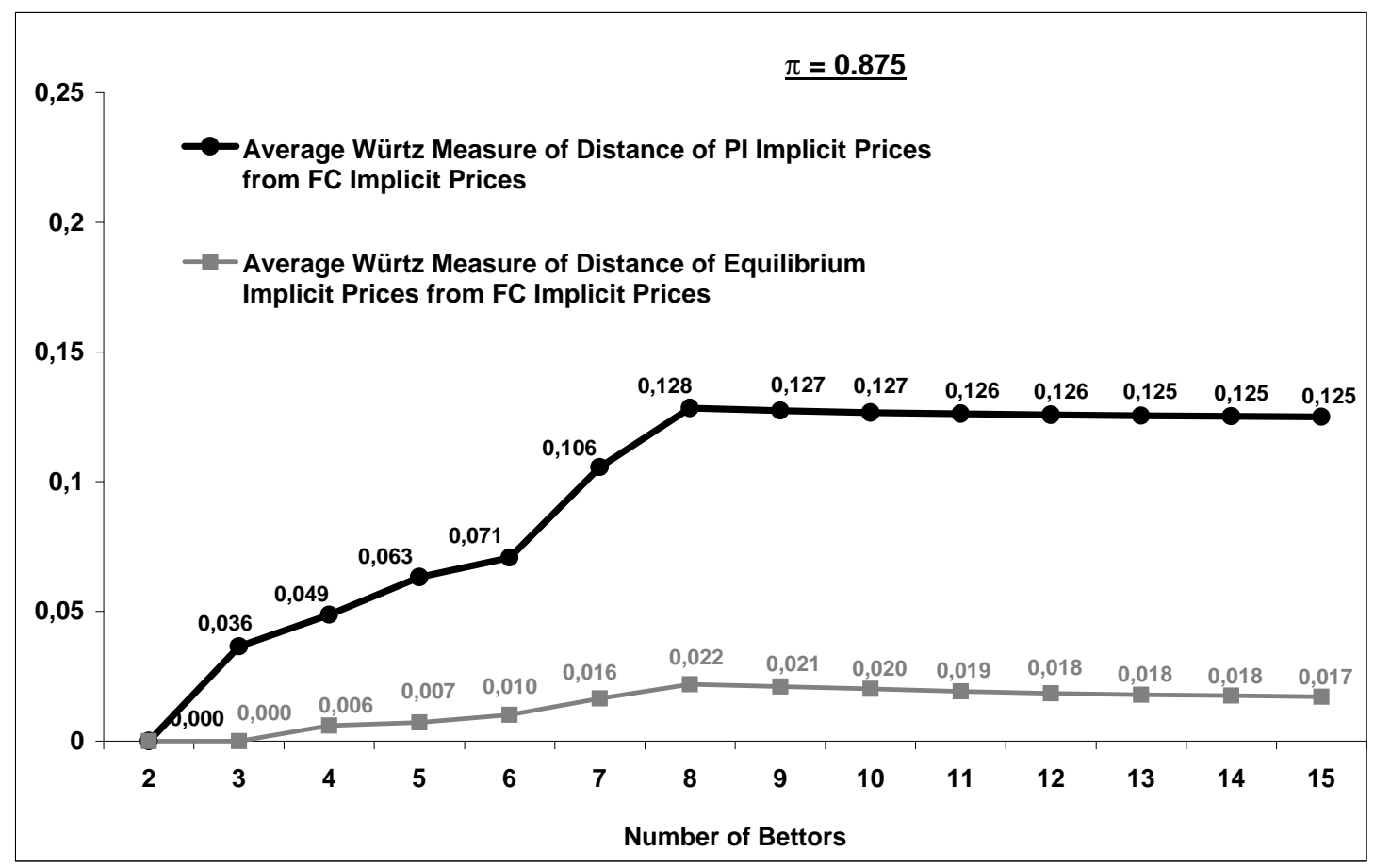

Figure 11: Evolution of Average Distances $V^{1}(\pi, n)$ and $V^{2}(\pi, n)$ up to Bettor 15 with $\pi=$ 0.875 . 


\section{Conclusion and Relation to Previous Work}

To the best of our knowledge, the analysis offered in this paper is the first to investigate betting behavior in parimutuel markets under asymmetric information. Not surprisingly, the temporal structure of the market drives the results. Though betting odds perfectly reflect all available information in the simultaneous move market game, non-informative behaviors emerge whatever the quality of players' information in a sequential parimutuel betting market. More precisely, we showed that a perfectly revealing equilibrium fails to exist with more than a dozen of bettors. We also illustrated the fact that extreme mispricing effects may occur. Such informational inefficiencies at various states of the world are due to herd behavior arising with intermediate qualities of information. Analyses of the average capacity of the sequential parimutuel mechanism to aggregate the disseminated information confirmed this former result and led to the opposite conclusion for extreme values of the quality of information.

In the following lines we first sketch a short bibliographic note on parimutuel betting games. Then we discuss the relationships which exist between our framework and the growing literature on social learning and information aggregation in sequential decision models with observable actions and asymmetric information.

Watanabe et al. (1994) are the first to analyze a game-theoretical model of parimutuel betting. They consider two horses and assumed that bettors' prior beliefs are inconsistent. In other words, agents agree to disagree, contrary to the Harsanyi doctrine. An ad hoc selection criterion is introduced to select a unique equilibrium which is regular in the sense that if there exists a player who bets on horse $A$ (respectively $B$ ) at an equilibrium, then players who have strictly larger (respectively smaller) probability for the win of this horse will always bet on it. Watanabe (1997) considers the same model but with a continuum of bettors. Ziegelmeyer, Broihanne, and Koessler (2001) and Feenay and King (2001) have independently developed a sequential betting game with consistent beliefs, a finite set of strategic bettors, and no withdrawing possibilities. ${ }^{14}$ By assuming symmetric information (and common prior beliefs on the winning chance of each horse), they show that the subgame perfect equilibrium is unique for almost all values of prior probabilities. In such an equilibrium, there is always an advantage to being an early mover and players betting first do not necessarily choose to bet on the horse with the highest probability to win. ${ }^{15}$ As shown by Propositions 8 and 9 of this paper, at least with two and three bettors, asymmetric information has "destroyed" this effect according to which early bettors might choose an action with a low ex ante expected gain. ${ }^{16}$ Besides the former remark, no relevant connection can be made between our results and the previous results obtained in the literature on parimutuel betting markets. On the contrary, our results can be linked to those obtained in traditional herding models, in models of sequential trade in financial markets, and in the literature on sequential voting.

It seems natural to draw a parallel between the analysis offered here and the literature on "information cascades". An information cascade can be defined as a choice sequence in which some agents act as if they ignored their private information and followed the choices made earlier in the sequence by other agents. ${ }^{17}$ In the pioneering herding models agents are only concerned with maximizing their own expected payoffs which cannot be directly affected by the actions of others. The complete absence of payoff interdependencies is obviously a

\footnotetext{
${ }^{14} \mathrm{~A}$ game-theoretical model under symmetric information where it is assumed that each bettor must spend his entire endowment on the race has also been analyzed by Chadha and Quandt (1996).

${ }^{15}$ This model was extended to the case where bettors have the possibility to withdraw by Koessler, Ziegelmeyer, and Broihanne (2002), by introducing a fraction of noise bettors into the game (see also Hurley and McDonough, 1995 and Terrell and Farmer, 1996 in simultaneous move betting games with transactions/information costs).

${ }^{16}$ Of course, this effect cannot arise with myopic bettors.

${ }^{17}$ See, e.g., Banerjee (1992), Bikhchandani, Hirshleifer, and Welch (1992), and Chamley and Gale (1994).
} 
strong assumption as in almost any game the payoff structure of an agent is affected by the other players' actions. In this respect, our work can be seen as an extension of rational herd behavior models where negative payoff externalities are considered. Even though long-run herding is impossible in sequential parimutuel betting markets, our results imply that herd behavior, at least in the short-run, is robust to the parimutuel mechanism.

Though most of the literature on rational herding assumes that prices for taking an action are fixed, a notable exception is Avery and Zemsky's (1998) model where the relationship between asset prices and herd behavior is investigated. Hence, as in sequential parimutuel betting markets under asymmetric information, along with informational externalities, payoff externalities arise through the addition of a price mechanism. The main differences between Avery and Zemsky's (1998) asset pricing model and parimutuel betting markets are the following. First, in Avery and Zemsky's (1998) model, the price is determined by a market maker according to his information about past trades, while in parimutuel betting markets the price mechanism is exogenous and ensures that average bettors' return is null or negative (negative if transaction costs are strictly positive). Second, in parimutuel betting markets the return of each player also depends on his expectation about the behavior of later participants. Hence, in such markets, the analysis is complicated by the fact that fully rational agents are concerned not only with learning from predecessors, but also with signaling to successors. ${ }^{18}$ This intractable difficulty was avoided in Section 5 by considering myopic agents. This latter framework is in fact very related to the one of Avery and Zemsky. More precisely, if we assume further that bettors do not recognize their impact on betting odds, i.e., if $u_{i}\left(s^{i}, \theta_{H}\right)=O_{H}\left(s^{i-1}\right)+1$ if $s_{i}=H$ and $u_{i}\left(s^{i}, \theta_{H}\right)=0$ if $s_{i} \neq H$, and if odds against each horse $H$ is replaced by $O_{H}\left(s^{i-1}\right)=\frac{1-\operatorname{Pr}\left(\theta_{H} \mid s^{i-1}\right)}{\operatorname{Pr}\left(\theta_{H} \mid s^{i-1}\right)}$, then the subjective probability of horse $H$ becomes equal to $\operatorname{Pr}\left(\theta_{H} \mid s^{i-1}\right)$, i.e., the price of the asset in Avery and Zemsky (1998). In that case, condition 10 on page 12 for bettor $i$ to follow his signal is replaced by $\mu_{i}\left(\theta_{H} \mid s^{i-1} ; q_{i}\right)>\operatorname{Pr}\left(\theta_{H} \mid s^{i-1}\right)$, which is satisfied iff $q_{i}=q^{H}$. Hence, bettors always follow their private signal. Our results contrast with Avery and Zemsky's (1998) ones since in Avery and Zemsky full revelation of information is obtained once an endogenous price is incorporated into the analysis. To obtain herd behavior, they have to consider information asymmetries between the market maker and traders. This is not necessary in parimutuel betting markets where the price mechanism is exogenous and independent of the types of participants. In particular, betting odds do not integrate bettors' decision rules.

Finally, our work can be related to the literature on sequential voting in the sense that this literature also extends information cascade models to a context in which an individual's payoff from taking an action is influenced by the actions of others. ${ }^{19}$ In particular, agents must consider the information their actions provide to agents later in the sequence. This literature also asks how sequential decisions differ from simultaneous decisions in terms of generated outcomes and information aggregation. However, betting markets are characterized by strong conflicting interests that we do not find in standard models of sequential voting. Additionally, only a player which is pivotal in voting models can affect the outcome, whereas all players always affect the payoffs of the others in parimutuel games. Our results, particularly the impossibility results concerning perfectly revealing equilibria, differ from those of this literature because our impossibility results do not require different qualities of information or more than two horses. This contrasts with the results obtained in sequential voting where there always exists a perfectly revealing equilibrium when there is only one quality and two candidates (see Theorem 1 in Dekel and Piccione, 2000).

\footnotetext{
${ }^{18}$ Dasgupta (2000) performs a similar analysis with the notable difference that he considers positive payoff externalities with an additional requirement of complete agreement on investment decisions. See also Corsetti, Dasgupta, Morris, and Shin (2001) in a 2-period model.

${ }^{19}$ See, e.g., Feddersen and Pesendorfer (1998), Dekel and Piccione (2000), and references therein.
} 


\section{Appendix}

Proof of Proposition 1. The strategy $\sigma_{i} \in \Sigma_{i}$ satisfying $\sigma_{i}\left(q^{H}\right)=\bar{H}$ for all $H \in$ $\{A, B\}$ is optimal for bettor $i$ if $U_{i}\left(\bar{H}, \sigma_{-i} \mid q^{H}\right) \geq U_{i}\left(H, \sigma_{-i} \mid q^{H}\right)$ for all $H$, i.e., $(1-$

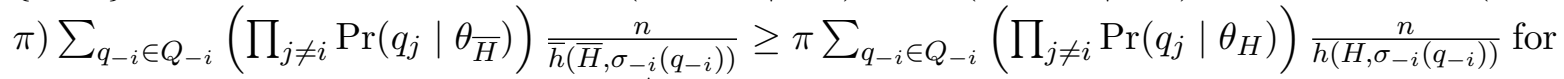
all $H$, which is impossible with $\pi>1 / 2$.

Proof of Proposition 2. To prove the existence of a fully informative equilibrium, let $i \in N$ and assume that $\sigma_{j}\left(q^{H}\right)=H$ for all $H \in\{A, B\}$ and $j \neq i$. Then, it is clear that

$$
\sum_{q_{-i} \in Q_{-i}}\left(\prod_{j \neq i} \operatorname{Pr}\left(q_{j} \mid \theta_{H}\right)\right) \frac{n}{h\left(H, \sigma_{-i}\left(q_{-i}\right)\right)}=\sum_{q_{-i} \in Q_{-i}}\left(\prod_{j \neq i} \operatorname{Pr}\left(q_{j} \mid \theta_{\bar{H}}\right)\right) \frac{n}{\bar{h}\left(\bar{H}, \sigma_{-i}\left(q_{-i}\right)\right)} .
$$

Therefore, for any $H \in\{A, B\}$, inequality $U_{i}\left(H, \sigma_{-i} \mid q^{H}\right) \geq U_{i}\left(\bar{H}, \sigma_{-i} \mid q^{H}\right)$ is satisfied if and only if $\operatorname{Pr}\left(\theta_{H} \mid q^{H}\right) \geq \operatorname{Pr}\left(\theta_{\bar{H}} \mid q^{H}\right)$, i.e., $\pi \geq 1-\pi$, which is satisfied since $\pi>1 / 2$.

To prove that the fully informative equilibrium is the unique symmetric equilibrium, remark that there are three other symmetric strategy profiles: (i) every bettor bets against his own private signal, (ii) every bettor bets on horse $A$, and (iii) every bettor bets on horse $B$. We prove that none of those strategy profiles form a Bayesian-Nash equilibrium.

(i) Directly from Proposition 1.

(ii) Let $\sigma_{i}\left(q^{H}\right)=A$ for all $H \in\{A, B\}$ and $i \in N$. Then, $U_{i}\left(B, \sigma_{-i} \mid q^{B}\right)=\operatorname{Pr}\left(\theta_{B} \mid q^{B}\right) \frac{n}{1}=$ $n \pi$ and $U_{i}\left(A, \sigma_{-i} \mid q^{B}\right)=\operatorname{Pr}\left(\theta_{A} \mid q^{B}\right) \frac{n}{n}=(1-\pi)<n \pi$. Hence, $\sigma$ is not an equilibrium.

(iii) Similar to (ii).

Proof of Proposition 3. Let $a$ be the number of bettors who bet on horse $A$ whatever their private signal, and let $b=n-a$ be the number of bettors who bet on horse $B$ whatever their private signal ( $a, b \neq 0$ from Proposition 2). The expected utility of each bettor $i$ who bets unconditionally on horse $A$ is given by

$$
\begin{aligned}
U_{i}\left(A, \sigma_{-i} \mid q^{A}\right) & =\operatorname{Pr}\left(\theta_{A} \mid q^{A}\right) \frac{n}{a}=\frac{n \pi}{a} \\
U_{i}\left(B, \sigma_{-i} \mid q^{A}\right) & =\operatorname{Pr}\left(\theta_{B} \mid q^{A}\right) \frac{n}{n-a+1}=\frac{n(1-\pi)}{n-a+1} \\
U_{i}\left(A, \sigma_{-i} \mid q^{B}\right) & =\operatorname{Pr}\left(\theta_{A} \mid q^{B}\right) \frac{n}{a}=\frac{n(1-\pi)}{a} \\
U_{i}\left(B, \sigma_{-i} \mid q^{B}\right) & =\operatorname{Pr}\left(\theta_{B} \mid q^{B}\right) \frac{n}{n-a+1}=\frac{n \pi}{n-a+1} .
\end{aligned}
$$

Similarly, the expected utility of each bettor $i$ who bets unconditionally on horse $B$ is given by

$$
\begin{aligned}
U_{i}\left(A, \sigma_{-i} \mid q^{A}\right) & =\operatorname{Pr}\left(\theta_{A} \mid q^{A}\right) \frac{n}{a+1}=\frac{n \pi}{a+1} \\
U_{i}\left(B, \sigma_{-i} \mid q^{A}\right) & =\operatorname{Pr}\left(\theta_{B} \mid q^{A}\right) \frac{n}{n-a}=\frac{n(1-\pi)}{n-a} \\
U_{i}\left(A, \sigma_{-i} \mid q^{B}\right) & =\operatorname{Pr}\left(\theta_{A} \mid q^{B}\right) \frac{n}{a+1}=\frac{n(1-\pi)}{a+1} \\
U_{i}\left(B, \sigma_{-i} \mid q^{B}\right) & =\operatorname{Pr}\left(\theta_{B} \mid q^{B}\right) \frac{n}{n-a}=\frac{n \pi}{n-a} .
\end{aligned}
$$

Thus, we have an equilibrium if $\frac{a}{n+1} \leq \pi \leq \frac{a+1}{n+1}$ and $\frac{b}{n+1} \leq \pi \leq \frac{b+1}{n+1}$. Hence, to have an equilibrium we must have $a=b=n / 2$, and thus $n$ is necessarily an even number. Additionally, 
we must have $\pi \leq \frac{n+2}{2 n+2}$. The fact that there exist exactly $C_{n}^{n / 2}$ non-informative equilibria is obvious. This completes the proof.

Proof of Proposition 4. Assume that $k<n$ bettors always follow their signal, a bettors always bet on horse $A$, and $b=n-a-k$ bettors always bet on horse $B$. Let $N_{K}$ be the set of bettors who follow their signals, $N_{A}$ the set of bettors who always bet on horse $A$, and $N_{B}$ the set of bettors who always bet on horse $B$. For each bettor $i \in N_{K}$ we have

$$
\begin{aligned}
& U_{i}\left(A, \sigma_{-i} \mid q^{A}\right)=n \pi\left(\sum_{j=0}^{k-1} \frac{C_{k-1}^{j} \pi^{j}(1-\pi)^{k-1-j}}{a+1+j}\right) \\
& U_{i}\left(B, \sigma_{-i} \mid q^{A}\right)=n(1-\pi)\left(\sum_{j=0}^{k-1} \frac{C_{k-1}^{j} \pi^{j}(1-\pi)^{k-1-j}}{b+1+j}\right) \\
& U_{i}\left(A, \sigma_{-i} \mid q^{B}\right)=n(1-\pi)\left(\sum_{j=0}^{k-1} \frac{C_{k-1}^{j} \pi^{j}(1-\pi)^{k-1-j}}{a+1+j}\right) \\
& U_{i}\left(B, \sigma_{-i} \mid q^{B}\right)=n \pi\left(\sum_{j=0}^{k-1} \frac{C_{k-1}^{j} \pi^{j}(1-\pi)^{k-1-j}}{b+1+j}\right) .
\end{aligned}
$$

For each bettor $i \in N_{A}$ we have

$$
\begin{aligned}
& U_{i}\left(A, \sigma_{-i} \mid q^{A}\right)=n \pi\left(\sum_{j=0}^{k} \frac{C_{k}^{j} \pi^{j}(1-\pi)^{k-j}}{a+j}\right) \\
& U_{i}\left(B, \sigma_{-i} \mid q^{A}\right)=n(1-\pi)\left(\sum_{j=0}^{k} \frac{C_{k}^{j} \pi^{j}(1-\pi)^{k-j}}{b+1+j}\right) \\
& U_{i}\left(A, \sigma_{-i} \mid q^{B}\right)=n(1-\pi)\left(\sum_{j=0}^{k} \frac{C_{k}^{j} \pi^{j}(1-\pi)^{k-j}}{a+j}\right) \\
& U_{i}\left(B, \sigma_{-i} \mid q^{B}\right)=n \pi\left(\sum_{j=0}^{k} \frac{C_{k}^{j} \pi^{j}(1-\pi)^{k-j}}{b+1+j}\right) .
\end{aligned}
$$

Finally, for each bettor $i \in N_{B}$ we have

$$
\begin{aligned}
& U_{i}\left(A, \sigma_{-i} \mid q^{A}\right)=n \pi\left(\sum_{j=0}^{k} \frac{C_{k}^{j} \pi^{j}(1-\pi)^{k-j}}{a+1+j}\right) \\
& U_{i}\left(B, \sigma_{-i} \mid q^{A}\right)=n(1-\pi)\left(\sum_{j=0}^{k} \frac{C_{k}^{j} \pi^{j}(1-\pi)^{k-j}}{b+j}\right) \\
& U_{i}\left(A, \sigma_{-i} \mid q^{B}\right)=n(1-\pi)\left(\sum_{j=0}^{k} \frac{C_{k}^{j} \pi^{j}(1-\pi)^{k-j}}{a+1+j}\right) \\
& U_{i}\left(B, \sigma_{-i} \mid q^{B}\right)=n \pi\left(\sum_{j=0}^{k} \frac{C_{k}^{j} \pi^{j}(1-\pi)^{k-j}}{b+j}\right) .
\end{aligned}
$$


It is easy to verify that each bettor $i \in N_{A}$ is rational only if $a<b+1$ and each bettor $i \in N_{B}$ is rational only if $b<a+1$, which implies that $b-1<a<b+1$, i.e., $a=b=\frac{n-k}{2}$. In this case, it is rational for each bettor $i \in N_{K}$ to follow his signal. Moreover, each bettor $i \in N_{H}$ rationally follows his signal when he receives the signal $q^{H}$. It remains to check under which conditions each bettor $i \in N_{H}$ bets on $H$ even when he receives the signal $q^{\bar{H}}$. From the expected utilities given above, this condition is satisfied if and only if

$$
\frac{\pi}{1-\pi} \leq \frac{\sum_{j=0}^{k} \frac{C_{k}^{j} \pi^{j}(1-\pi)^{k-j}}{a+j}}{\sum_{j=0}^{k} \frac{C_{k}^{j} \pi^{j}(1-\pi)^{k-j}}{a+1+j}}=\frac{\sum_{j=0}^{k} \frac{\left(\frac{\pi}{1-\pi}\right)^{j}}{j !(k-j) !(a+j)}}{\sum_{j=0}^{k} \frac{\left(\frac{\pi}{1-\pi}\right)^{j}}{j !(k-j) !(a+j+1)}}
$$

where $a=\frac{n-k}{2}$ and $0 \leq k<n$. The fact that there are exactly $C_{n}^{k} \times C_{n-k}^{\frac{n-k}{2}}$ partially informative equilibria with $k$ informative bets is obvious.

Proof of Lemma 1. Let $f(k, n, \pi)=\frac{\pi}{1-\pi}-\frac{\sum_{j=0}^{k} \frac{C_{k}^{j} \pi^{j}(1-\pi)^{k-j}}{\frac{n-k}{2}+j}}{\sum_{j=0}^{k} \frac{C_{k}^{j} \pi^{j}(1-\pi)^{k-j}}{\frac{n-k}{2}+1+j}}$. We have to show that $f(k, n, \pi) \leq 0$ is equivalent to $g(k, n, \pi) \leq 0$. Let $\left.x=\frac{\pi}{1-\pi} \in\right] 1,+\infty\left[\right.$ and $a=\frac{n-k}{2}$. We have $f(k, n, \pi) \leq 0 \Leftrightarrow x \leq \frac{\sum_{j=0}^{k} \frac{C_{k}^{j} x^{j}}{a+j}}{\sum_{j=0}^{k} \frac{C_{k}^{j} x^{j}}{a+j+1}} \Leftrightarrow \sum_{j=-1}^{k} \frac{C_{k}^{j} x^{j+1}}{a+j+1} \leq \sum_{j=0}^{k+1} \frac{C_{k}^{j} x^{j}}{a+j}$, since by convention $C_{k}^{-1}=C_{k}^{k+1}=0$. The last inequality is equivalent to $\sum_{j=0}^{k+1} \frac{x^{j}}{a+j}\left(C_{k}^{j}-C_{k}^{j-1}\right) \geq 0$. Using the definition of the binomial coefficient, it is not difficult to show that $C_{k}^{j}-C_{k}^{j-1}=$ $C_{k+1}^{j} \frac{k+1-2 j}{k+1}$. Substituting this value into the last inequality, we get $\sum_{j=0}^{k+1} \frac{x^{j}}{a+j} C_{k+1}^{j} \frac{k+1-2 j}{k+1} \geq$ $0 \Leftrightarrow \sum_{j=0}^{k+1} x^{j} C_{k+1}^{j} \frac{k+1-2 j}{\left(\frac{n-k}{2}+j\right)(k+1)} \geq 0 \Leftrightarrow \sum_{j=0}^{k+1} x^{j} C_{k+1}^{j} \frac{k+1-2 j}{n-k+2 j} \geq 0 \Leftrightarrow g(k, n, \pi) \leq 0$.

Proof of Lemma 2. It is equivalent to prove this property for $f(k, n, \pi)$. We have $f(k, n, 1 / 2)=1-\frac{\sum_{j=0}^{k} \frac{1}{j !(k-j) !\left(\frac{n-k}{2}+j\right)}}{\sum_{j=0}^{k} \frac{1}{j !(k-j) !\left(\frac{n-k}{2}+j+1\right)}}<0$. Moreover, $\lim _{\pi \rightarrow 1^{-}} \frac{\sum_{j=0}^{k} \frac{C_{k}^{j} \pi^{j}(1-\pi)^{k-j}}{\frac{n-k}{2}+j}}{\sum_{j=0}^{k} \frac{C_{k}^{j} \pi^{j}(1-\pi)^{k-j}}{\frac{n-k}{2}+1+j}}=\frac{\frac{n-k}{2}+k+1}{\frac{n-k}{2}+k}$, and $\lim _{\pi \rightarrow 1^{-}} \frac{\pi}{1-\pi}=+\infty$, which implies that $\lim _{\pi \rightarrow 1^{-}} f(k, n, \pi)>0$ for all $n$ and $k$. Hence, there exists $\left.\pi, \pi^{\prime} \in\right] 1 / 2,1\left[\right.$ such that $f(k, n, \pi)<0$ and $f\left(k, n, \pi^{\prime}\right)>0$.

Proof of Lemma 3. We have to show that $\frac{\partial g(k, n, \pi)}{\partial \pi}>0$ for all $\left.\pi \in\right] 1 / 2,1\left[\right.$. Let $x=\frac{\pi}{1-\pi} \in$ ] $1,+\infty\left[\right.$. We have $\frac{\partial g(k, n, \pi)}{\partial \pi}>0 \Leftrightarrow \sum_{j=0}^{k+1} j x^{j-1} C_{k+1}^{j} \frac{k-2 j+1}{k-2 j-n}>0$. Assume that $k$ is an even number. Then, the last inequality is equivalent to

$$
\begin{aligned}
& \sum_{j=0}^{k / 2} j x^{j-1} C_{k+1}^{j} \frac{k-2 j+1}{n+2 j-k}<\sum_{j=(k / 2)+1}^{k+1} j x^{j-1} C_{k+1}^{j} \frac{2 j-k-1}{n+2 j-k} \\
\Leftrightarrow & \sum_{j=0}^{k / 2} j x^{j-1} C_{k+1}^{j} \frac{k-2 j+1}{n+2 j-k}<\sum_{j=0}^{k / 2}(k+1-j) x^{k-j} C_{k+1}^{k+1-j} \frac{2(k+1-j)-k-1}{n+2(k+1-j)-k} \\
\Leftrightarrow & \sum_{j=0}^{k / 2} j x^{j-1} C_{k+1}^{j} \frac{k-2 j+1}{n+2 j-k}<\sum_{j=0}^{k / 2}(k+1-j) x^{k-j} C_{k+1}^{j} \frac{k-2 j+1}{n-2 j+k+2},
\end{aligned}
$$

because $C_{k+1}^{j}=C_{k+1}^{k+1-j}$. To prove the last inequality, it is sufficient to show that for all $j=0,1, \ldots, k / 2$ we have $j x^{j-1} C_{k+1}^{j} \frac{k-2 j+1}{n+2 j-k}<(k+1-j) x^{k-j} C_{k+1}^{j} \frac{k-2 j+1}{n-2 j+k+2}$. Since $x>1 \Rightarrow$ 
$x^{j-1}<x^{k-j}$ for all $j=0,1, \ldots, k / 2$, it is sufficient to show that $j \frac{k-2 j+1}{n+2 j-k} \leq(k+1-j) \frac{k-2 j+1}{n-2 j+k+2}$ or, equivalently, $k+1-2 j \geq 0$, which is satisfied for all $j=0,1, \ldots, k / 2$. Now, we assume that $k$ is an odd number. Then, we have

$$
\begin{aligned}
& \sum_{j=0}^{(k-1) / 2} j x^{j-1} C_{k+1}^{j} \frac{k-2 j+1}{n+2 j-k}<\sum_{j=(k+1) / 2}^{k+1} j x^{j-1} C_{k+1}^{j} \frac{2 j-k-1}{n+2 j-k} \\
\Leftrightarrow & \sum_{j=0}^{(k-1) / 2} j x^{j-1} C_{k+1}^{j} \frac{k-2 j+1}{n+2 j-k}<\sum_{j=(k+3) / 2}^{k+1} j x^{j-1} C_{k+1}^{j} \frac{2 j-k-1}{n+2 j-k} \\
\Leftrightarrow & \sum_{j=0}^{(k-1) / 2} j x^{j-1} C_{k+1}^{j} \frac{k-2 j+1}{n+2 j-k}<\sum_{j=0}^{(k-1) / 2}(k+1-j) x^{k-j} C_{k+1}^{k+1-j} \frac{k-2 j+1}{n-2 j+k+2} .
\end{aligned}
$$

The rest of the proof is as before.

Proof of Proposition 5. Directly from Lemmas 2 and 3.

Proof of Lemma 4. Directly from the expected utility of Equation (5) on page 8.

Proof of Lemma 5. From Bayes rule (Equation (6) on page 8) with $J_{j}\left(H \mid s^{j-1}\right)=\left\{q^{H}\right\}$ (because every player follows his signal by assumption), after some simplifications.

Proof of Proposition 6. Let $(\sigma, \mu)$ be a perfectly revealing equilibrium. This implies that the last bettor always follows his signal: $\sigma_{n}\left(s^{n-1} ; q^{H}\right)=H$ for all $s^{n-1} \in S^{n-1}$. In particular, (i) $\sigma_{n}\left(\bar{H}, \ldots, \bar{H} ; q^{H}\right)=H$ and (ii) $\sigma_{n}\left(H, \ldots, H ; q^{H}\right)=H$.

(i) From Lemmas 4 and 5 we have $(h=0): \mu_{n}\left(\theta_{H} \mid s^{n-1} ; q^{H}\right) \geq \frac{h\left(s^{n-1}\right)+1}{n+1} \Leftrightarrow \frac{\pi^{2-n}}{\pi^{2-n}+(1-\pi)^{2-n}}$ $\geq \frac{1}{n+1} \Leftrightarrow \frac{n^{1 /(n-2)}}{1+n^{1 /(n-2)}} \geq \pi$.

(ii) From Lemmas 4 and 5 we have $(h=n-1): \mu_{n}\left(\theta_{H} \mid s^{n-1} ; q^{H}\right) \geq \frac{h\left(s^{n-1}\right)+1}{n+1} \Leftrightarrow$ $\frac{\pi^{n}}{\pi^{n}+(1-\pi)^{n}} \geq \frac{n}{n+1} \Leftrightarrow \pi \geq \frac{n^{1 / n}}{1+n^{1 / n}}$. This completes the proof.

Proof of Proposition 7. Assume that $\sigma_{-i}$ is perfectly revealing, i.e., all bettors other than $i$ follow their own private signal. Let $a=a\left(s^{i-1}\right)$ and consider player $i$ with the signal $q^{A}$ (the same conditions are obtained when he gets the signal $\left.q^{B}\right)$. From Equation (5), his utility can be written $U_{i}\left(A \mid s^{i-1} ; q^{A}\right)=n \mu_{i}\left(\theta_{A} \mid s^{i-1} ; q^{A}\right) \sum_{q_{i+} \in Q_{i^{+}}} \operatorname{Pr}\left(q_{i^{+}} \mid \theta_{A}\right) \frac{1}{a+1+\left|\left\{j>i: q_{j}=q^{A}\right\}\right|}$, since every bettor $j>i$ follows his private signal. Moreover from Lemma 5 we have $U_{i}\left(A \mid s^{i-1} ; q^{A}\right)=$ $n \frac{\pi^{2 a+2-i}}{\pi^{2 a+2-i}+(1-\pi)^{2 a+2-i}} \sum_{q_{i+} \in Q_{i^{+}}} \operatorname{Pr}\left(q_{i^{+}} \mid \theta_{A}\right) \frac{1}{a+1+\alpha\left(q_{i^{+}}\right)}$, where $\alpha\left(q_{i^{+}}\right)=\left|\left\{j>i: q_{j}=q^{A}\right\}\right|$. Similarly, $U_{i}\left(B \mid s^{i-1} ; q^{A}\right)=n \frac{(1-\pi)^{2 a+2-i}}{\pi^{2 a+2-i}+(1-\pi)^{2 a+2-i}} \sum_{q_{i+} \in Q_{i^{+}}} \operatorname{Pr}\left(q_{i^{+}} \mid \theta_{B}\right) \frac{1}{(i-a)+\beta\left(q_{i^{+}}\right)}$, where $\beta\left(q_{i^{+}}\right)=\left|\left\{j>i: q_{j}=q^{B}\right\}\right|$. Hence, $U_{i}\left(A \mid s^{i-1} ; q^{A}\right) \geq U_{i}\left(B \mid s^{i-1} ; q^{A}\right)$ if and only if $\left(\frac{\pi}{1-\pi}\right)^{2 a+2-i} \geq \frac{\sum_{q_{i+} \in Q_{i+}} \frac{\operatorname{Pr}\left(q_{i+} \mid \theta_{B}\right)}{(i-a)+\beta\left(q_{i+}\right)}}{\sum_{q_{i} \in Q_{i+}} \frac{\operatorname{Pr}\left(q_{i}+\mid \theta A\right)}{a+1+\alpha\left(q_{i}\right)}} \equiv \frac{x_{i}(B)}{x_{i}(A)}$. For all $j \in\{0,1, \ldots, n-i\},\left\{q_{i^{+}} \in Q_{i^{+}}\right.$: $\left.\alpha\left(q_{i^{+}}\right)=j\right\}$ is the set of vectors of signals of bettors after $i$ such that exactly $j$ signals favor horse $A$. Clearly, we have $\left|\left\{q_{i^{+}} \in Q_{i^{+}}: \alpha\left(q_{i^{+}}\right)=j\right\}\right|=C_{n-i}^{j}$. Moreover, for all $q_{i^{+}} \in\left\{q_{i^{+}} \in Q_{i^{+}}: \alpha\left(q_{i^{+}}\right)=j\right\}, \operatorname{Pr}\left(q_{i^{+}} \mid \theta_{A}\right)=\pi^{j}(1-\pi)^{n-i-j}$. Therefore, we get $x_{i}(A)=\sum_{j=0}^{n-i} \frac{C_{n-i}^{j} \pi^{j}(1-\pi)^{n-i-j}}{a+1+j}$ and, similarly, $x_{i}(B)=\sum_{j=0}^{n-i} \frac{C_{n-i}^{j} \pi^{j}(1-\pi)^{n-i-j}}{i-a+j}$. To summarize, if $\sigma_{-i}$ is a profile of perfectly revealing strategies, then player $i$ follows his signal if $\left(\frac{\pi}{1-\pi}\right)^{2 a+2-i} \geq \frac{\sum_{j=0}^{n-i} \frac{C_{n-i}^{j} \pi^{j}(1-\pi)^{n-i-j}}{i-a+j}}{\sum_{j=0}^{n-i} \frac{C_{n-i}^{j} \pi^{j}(1-\pi)^{n-i-j}}{a+1+j}}$. Hence, a perfectly revealing equilibrium is obtained if the last inequality is satisfied for all $i \in N$ and $a \in\{0,1, \ldots, i-1\}$. 
Proof of Proposition 8. To prove the proposition we analyze all possible strategies of the first bettor and we examine the associated best response of the second one depending on his possible outside equilibrium beliefs. The first bettor has three types of possible strategies: i) He follows his signal; ii) he bets against his signal; iii) he bets non-informatively.

i) The First Bettor Follows his Signal. We check for the existence of an equilibrium in which the first bettor always follows his signal, i.e., $\sigma_{1}\left(q^{A}\right)=A$ and $\sigma_{1}\left(q^{B}\right)=B$. In this case, Bayes' rule applies everywhere and we obtain the following beliefs for the second bettor: $\mu_{2}\left(\theta_{A} \mid A ; q^{A}\right)=\mu_{2}\left(\theta_{B} \mid B ; q^{B}\right)=\frac{\pi^{2}}{\pi^{2}+(1-\pi)^{2}}, \mu_{2}\left(\theta_{A} \mid A ; q^{B}\right)=\mu_{2}\left(\theta_{B} \mid B ; q^{A}\right)=\mu_{2}\left(\theta_{A} \mid\right.$ $\left.B ; q^{A}\right)=\mu_{2}\left(\theta_{B} \mid A ; q^{B}\right)=1 / 2$, and $\mu_{2}\left(\theta_{A} \mid B ; q^{B}\right)=\mu_{2}\left(\theta_{B} \mid A ; q^{A}\right)=\frac{(1-\pi)^{2}}{\pi^{2}+(1-\pi)^{2}}$. From those beliefs, we can compute the expected utilities of bettor 2 depending on his signal, the observed history, and his betting choice. We easily obtain $U_{2}\left(A \mid A ; q^{A}\right)=U_{2}\left(B \mid B ; q^{B}\right)=\frac{\pi^{2}}{\pi^{2}+(1-\pi)^{2}}$, $U_{2}\left(A \mid B ; q^{B}\right)=U_{2}\left(B \mid A ; q^{A}\right)=\frac{2(1-\pi)^{2}}{\pi^{2}+(1-\pi)^{2}}, U_{2}\left(A \mid A ; q^{B}\right)=U_{2}\left(B \mid B ; q^{A}\right)=\frac{1}{2}$, and $U_{2}\left(A \mid B ; q^{A}\right)=U_{2}\left(B \mid A ; q^{B}\right)=1$. Therefore, for any $H \in\{A, B\}, \sigma_{2}\left(\bar{H} ; q^{H}\right)=H$ and

$$
\sigma_{2}\left(H ; q^{H}\right)= \begin{cases}H & \text { if } \pi>\frac{\sqrt{2}}{1+\sqrt{2}}=2-\sqrt{2} \simeq 0.586 \\ \bar{H} & \text { if } \pi<\frac{\sqrt{2}}{1+\sqrt{2}} .\end{cases}
$$

It remains to verify if bettor 1 is rational for all $\pi>1 / 2$ when bettor 2 uses the above strategy. Given $\sigma_{2}$, we obtain the following expected utilities:

$$
\begin{aligned}
U_{1}\left(A, \sigma_{2} \mid q^{A}\right) & =\mu_{1}\left(\theta_{A} \mid q^{A}\right) \sum_{q_{2} \in Q_{2}} \operatorname{Pr}\left(q_{2} \mid \theta_{A}\right)\left(1+O_{A}\left(A, \sigma_{2}\left(A ; q_{2}\right)\right)\right) \\
& =\pi \sum_{q_{2} \in Q_{2}} \operatorname{Pr}\left(q_{2} \mid \theta_{A}\right) \frac{2}{a\left(A, \sigma_{2}\left(A ; q_{2}\right)\right)} \\
& = \begin{cases}\pi(2-\pi) & \text { if } \pi>\frac{\sqrt{2}}{1+\sqrt{2}} \\
2 \pi & \text { otherwise. }\end{cases}
\end{aligned}
$$

Similarly,

$$
\begin{aligned}
& U_{1}\left(A, \sigma_{2} \mid q^{A}\right)=U_{1}\left(B, \sigma_{2} \mid q^{B}\right)= \begin{cases}\pi(2-\pi) & \text { if } \pi>\frac{\sqrt{2}}{1+\sqrt{2}} \\
2 \pi & \text { otherwise }\end{cases} \\
& U_{1}\left(B, \sigma_{2} \mid q^{A}\right)=U_{1}\left(A, \sigma_{2} \mid q^{B}\right)= \begin{cases}(1-\pi)(2-\pi) & \text { if } \pi>\frac{\sqrt{2}}{1+\sqrt{2}} \\
2(1-\pi) & \text { otherwise. }\end{cases}
\end{aligned}
$$

We get $\sigma_{1}\left(q^{H}\right)=H$, for any $\pi>1 / 2$. Hence, player 1 follows his own private signal.

ii) The First Bettor Bets Against his Signal. Assume that $\sigma_{2}\left(q^{H}\right)=\bar{H}$. Bayes' rule applies everywhere and we obtain the following beliefs for bettor 2: $\mu_{2}\left(\theta_{H} \mid \bar{H} ; q^{H}\right)=\frac{\pi^{2}}{\pi^{2}+(1-\pi)^{2}}$, $\mu_{2}\left(\theta_{H} \mid H ; q^{H}\right)=1 / 2$, and $\mu_{2}\left(\theta_{H} \mid H ; q^{\bar{H}}\right)=\frac{(1-\pi)^{2}}{\pi^{2}+(1-\pi)^{2}}$. Bettor 2's expected utility is then $U_{2}\left(H \mid H ; q^{\bar{H}}\right)=\frac{(1-\pi)^{2}}{\pi^{2}+(1-\pi)^{2}}, U_{2}\left(H \mid H ; q^{H}\right)=\frac{1}{2}, U_{2}\left(H \mid \bar{H} ; q^{\bar{H}}\right)=1$, and $U_{2}\left(H \mid \bar{H} ; q^{H}\right)=$ $\frac{2 \pi^{2}}{\pi^{2}+(1-\pi)^{2}}$. It follows immediately that the second bettor always adopts a contrarian behavior, whatever his private signal: $\sigma_{2}\left(H, q_{2}\right)=\bar{H}$ for all $q_{2} \in Q_{2}$. Now consider bettor 1 . It is easy to see that $U_{1}\left(H \mid q^{H}\right)=2 \pi$ and $U_{1}\left(\bar{H} \mid q^{H}\right)=2(1-\pi)<2 \pi$. Indeed, since the second bettor always bets on the other horse, the odds against the horse chosen by the first bettor is 
always equal to 2 . Hence, there is no equilibrium where the first bettor always bets against his private signal.

iii) The First Bettor Bets Non-Informatively. Assume that $\sigma_{1}\left(q_{1}\right)=H \in\{A, B\}$ for all $q_{1} \in Q_{1}$. The second bettor cannot apply Bayes' rule when he observes that the first bettor has bet on horse $\bar{H}$. When he observes $H$, he keeps his posterior beliefs. We obtain $\sigma_{2}\left(H ; q^{\bar{H}}\right)=\bar{H}$ and

$$
\sigma_{2}\left(H ; q^{H}\right)= \begin{cases}H & \text { if } \pi>2 / 3 \\ \bar{H} & \text { if } \pi<2 / 3\end{cases}
$$

To determine bettor 2's optimal action when he has observed a first bet on horse $\bar{H}$ we have to specify outside equilibrium beliefs. By restricting our attention to consistent beliefs (in the sense of Kreps and Wilson, 1982), the following conditions must be satisfied: $\mu_{2}\left(\theta_{H} \mid \bar{H} ; q^{H}\right) \in\left[1 / 2, \frac{\pi^{2}}{\pi^{2}+(1-\pi)^{2}}\right]$, and $\mu_{2}\left(\theta_{H} \mid \bar{H} ; q^{\bar{H}}\right) \in\left[\frac{(1-\pi)^{2}}{\pi^{2}+(1-\pi)^{2}}, 1 / 2\right]$. Hence, $U_{2}(H \mid$ $\left.\bar{H} ; q^{H}\right)=2 \mu_{2}\left(\theta_{H} \mid \bar{H} ; q^{H}\right) \geq 1$ and $U_{2}\left(\bar{H} \mid \bar{H} ; q^{H}\right)=1-\mu_{2}\left(\theta_{H} \mid \bar{H} ; q^{H}\right) \leq 1 / 2$, which implies that $\sigma_{2}\left(\bar{H} ; q^{H}\right)=H$. We obtain the following expected utilities for bettor 1 :

$$
\begin{array}{llrl}
U_{1}(H \mid \bar{H}) & =2(1-\pi)(\pi / 2+(1-\pi))=(1-\pi)(2-\pi) & & \text { if } \pi>2 / 3 \\
U_{1}(H \mid \bar{H}) & =2(1-\pi)(\pi+(1-\pi))=2(1-\pi) & & \text { if } \pi<2 / 3,
\end{array}
$$

and

$$
\begin{array}{ll}
U_{1}(\bar{H} \mid \bar{H})=2 \pi(\pi / 2+(1-\pi))=\pi(2-\pi) & \text { if } \sigma_{2}\left(\bar{H} ; q^{\bar{H}}\right)=\bar{H} \\
U_{1}(\bar{H} \mid \bar{H})=2 \pi(\pi+(1-\pi))=2 \pi & \text { if } \sigma_{2}\left(\bar{H} ; q^{\bar{H}}\right)=H .
\end{array}
$$

It is clear that if $\pi>2 / 3$ or $\sigma_{2}\left(\bar{H} ; q^{\bar{H}}\right)=H$, then bettor 1 deviates by following his signal $q^{\bar{H}}$. Hence, assume that $\pi<2 / 3$ and $\sigma_{2}\left(\bar{H} ; q^{\bar{H}}\right)=\bar{H}$. This last strategy is used by bettor 2 if $U_{2}\left(\bar{H} \mid \bar{H} ; q^{\bar{H}}\right) \geq U_{2}\left(H \mid \bar{H} ; q^{\bar{H}}\right)$. Since $U_{2}\left(\bar{H} \mid \bar{H} ; q^{\bar{H}}\right) \leq \frac{\pi^{2}}{\pi^{2}+(1-\pi)^{2}}$ and $U_{2}\left(H \mid \bar{H} ; q^{\bar{H}}\right) \geq$ $\frac{2(1-\pi)^{2}}{\pi^{2}+(1-\pi)^{2}}$, we must have $\pi \geq \frac{\sqrt{2}}{1+\sqrt{2}}$. Thereafter, note that $U_{1}(H \mid \bar{H}) \geq U_{1}(\bar{H} \mid \bar{H})$ if and only if $\pi \leq \frac{\sqrt{2}}{1+\sqrt{2}}$. Thus, whenever $\pi \neq \frac{\sqrt{2}}{1+\sqrt{2}}$, the first bettor never uses a non-informative strategy.

Proof of Proposition 9. First note that if the first and the second bettors follow their signals, then there is no outside equilibrium beliefs to specify. Beliefs of the second bettor are the same as in the 2-bettor case. Beliefs of the third bettor are given by $\mu_{3}\left(\theta_{A} \mid A, A ; q^{A}\right)=$ $\frac{\pi^{3}}{\pi^{3}+(1-\pi)^{3}}, \mu_{3}\left(\theta_{A} \mid B, B ; q^{B}\right)=\frac{(1-\pi)^{3}}{\pi^{3}+(1-\pi)^{3}}, \mu_{3}\left(\theta_{A} \mid A, A ; q^{B}\right)=\mu_{3}\left(\theta_{A} \mid A, B ; q^{A}\right)=\mu_{3}\left(\theta_{A} \mid\right.$ $\left.B, A ; q^{A}\right)=\pi, \mu_{3}\left(\theta_{A} \mid B, B ; q^{A}\right)=\mu_{3}\left(\theta_{A} \mid B, A ; q^{B}\right)=\mu_{3}\left(\theta_{A} \mid A, B ; q^{B}\right)=1-\pi$. From those beliefs we can compute the expected utilities of the third bettor, and then his optimal strategy: $\sigma_{3}\left(H, \bar{H} ; q^{H}\right)=\sigma_{3}\left(\bar{H}, H ; q^{H}\right)=H$, for all $\pi$, and

$$
\begin{aligned}
& \sigma_{3}\left(H, H ; q^{H}\right)= \begin{cases}H & \text { if } \pi>\frac{\sqrt[3]{3}}{1+\sqrt[3]{3}} \simeq 0.591 \\
\bar{H} & \text { if } \pi<\frac{\sqrt[3]{3}}{1+\sqrt[3]{3}}\end{cases} \\
& \sigma_{3}\left(H, H ; q^{\bar{H}}\right)= \begin{cases}H & \text { if } \pi>\frac{3}{4} \\
\bar{H} & \text { if } \pi<\frac{3}{4}\end{cases}
\end{aligned}
$$


Thereafter, we get the expected utilities of the second bettor:

$$
\begin{aligned}
& U_{2}\left(A, \sigma_{3} \mid A ; q^{A}\right)=U_{2}\left(B, \sigma_{3} \mid B ; q^{B}\right)= \begin{cases}\frac{\pi^{2}}{\pi^{2}+(1-\pi)^{2}} & \text { if } \pi>3 / 4 \\
\frac{\pi^{2}}{\pi^{2}+(1-\pi)^{2}} \frac{3-\pi}{2} & \text { if } 3 / 4>\pi>\frac{\sqrt[3]{3}}{1+\sqrt[3]{3}} \\
\frac{\pi^{2}}{\pi^{2}+(1-\pi)^{2}} \frac{3}{2} & \text { if } \pi<\frac{\sqrt[3]{3}}{1+\sqrt[3]{3}}\end{cases} \\
& U_{2}\left(B, \sigma_{3} \mid A ; q^{A}\right)=U_{2}\left(A, \sigma_{3} \mid B ; q^{B}\right)=\frac{(1-\pi)^{2}}{\pi^{2}+(1-\pi)^{2}} \frac{3(2-\pi)}{2} \quad \text { for all } \pi \\
& U_{2}\left(A, \sigma_{3} \mid A ; q^{B}\right)=U_{2}\left(B, \sigma_{3} \mid B ; q^{A}\right)= \begin{cases}\frac{1}{2} & \text { if } \pi>3 / 4 \\
\frac{3-\pi}{4} & \text { if } 3 / 4>\pi>\frac{\sqrt[3]{3}}{1+\sqrt[3]{3}} \\
\frac{3}{4} & \text { if } \pi<\frac{\sqrt[3]{3}}{1+\sqrt[3]{3}}\end{cases} \\
& U_{2}\left(A, \sigma_{3} \mid B ; q^{A}\right)=U_{2}\left(B, \sigma_{3} \mid A ; q^{B}\right)=\frac{3(2-\pi)}{4} \text { for all } \pi .
\end{aligned}
$$

We now turn to the optimal strategy of the second bettor. From the previous expected utilities it is clear that $U_{2}\left(H, \sigma_{3} \mid \bar{H} ; q^{H}\right) \geq U_{2}\left(\bar{H}, \sigma_{3} \mid \bar{H} ; q^{H}\right)$ for all $H \in\{A, B\}$ and $\pi \geq 1 / 2$. Hence, if the private signal of the second bettor contradicts the private signal of the first one, then the second bettor always follows his private signal (and thus bets against the first bettor's choice). Otherwise, we have:

If $\pi \geq 3 / 4$, then $U_{2}\left(A, \sigma_{3} \mid A ; q^{A}\right) \geq U_{2}\left(B, \sigma_{3} \mid A ; q^{A}\right) \Leftrightarrow \pi^{2} \geq(3 / 2)(1-\pi)^{2}(2-\pi) \Leftrightarrow$

$$
\pi \geq \frac{1}{9}\left(10-\frac{35}{(27 \sqrt{179}-296)^{1 / 3}}+(27 \sqrt{179}-296)^{1 / 3}\right) \simeq 0.592<3 / 4 .
$$

$\pi) \Leftrightarrow$

If $3 / 4 \geq \pi \geq \frac{\sqrt[3]{3}}{1+\sqrt[3]{3}}$, then $U_{2}\left(A, \sigma_{3} \mid A ; q^{A}\right) \geq U_{2}\left(B, \sigma_{3} \mid A ; q^{A}\right) \Leftrightarrow \pi^{2}(3-\pi) \geq 3(1-\pi)^{2}(2-$

$$
\pi \geq \frac{1}{2}\left(3-\frac{1}{(\sqrt{37}-6)^{1 / 3}}+(\sqrt{37}-6)^{1 / 3}\right) \simeq 0.571<\frac{\sqrt[3]{3}}{1+\sqrt[3]{3}} .
$$

If $\pi \leq \frac{\sqrt[3]{3}}{1+\sqrt[3]{3}}$, then $U_{2}\left(A, \sigma_{3} \mid A ; q^{A}\right) \geq U_{2}\left(B, \sigma_{3} \mid A ; q^{A}\right) \Leftrightarrow \pi^{2} \geq(1-\pi)^{2}(2-\pi) \Leftrightarrow$

$$
\pi \geq 1-2\left(\frac{2}{3(\sqrt{177}-9)}\right)^{1 / 3}+\frac{\left(\frac{1}{2}(\sqrt{177}-9)\right)^{1 / 3}}{3^{2 / 3}} \simeq 0.547<\frac{\sqrt[3]{3}}{1+\sqrt[3]{3}} .
$$

Thus, bettor 2 always follows his signal if $\pi \geq 1-2\left(\frac{2}{3(\sqrt{177}-9)}\right)^{1 / 3}+\frac{\left(\frac{1}{2}(\sqrt{177}-9)\right)^{1 / 3}}{3^{2 / 3}} \simeq 0.547$. Otherwise, he goes against the trend.

We assume in the following lines that $\pi \geq 0.547$ (otherwise, the equilibrium we are currently analyzing breaks down). Recalling that we assume that the second bettor always follows his own private signal and that the third bettor acts according to his strategy $\sigma_{3}$ found before (which depends on $\pi$ ), we get (after some calculations) the following expected utilities of the first bettor:

$$
\begin{aligned}
U_{1}\left(A, \sigma_{2}, \sigma_{3} \mid q^{A}\right)=U_{1}\left(B, \sigma_{2}, \sigma_{3} \mid q^{B}\right)= \\
\qquad \begin{array}{ll}
\frac{\pi}{2}\left(2 \pi^{2}+5 \pi(1-\pi)+6(1-\pi)^{2}\right) & \text { if } \pi>3 / 4 \\
\pi\left(\pi^{2}+3 \pi(1-\pi)+3(1-\pi)^{2}\right) & \text { if } 3 / 4>\pi>\frac{\sqrt[3]{3}}{1+\sqrt[3]{3}} \\
\frac{3 \pi}{2}\left(\pi^{2}+2 \pi(1-\pi)+2(1-\pi)^{2}\right) & \text { if } \pi<\frac{\sqrt[3]{3}}{1+\sqrt[3]{3}},
\end{array}
\end{aligned}
$$




$$
\begin{aligned}
U_{1}\left(B, \sigma_{2}, \sigma_{3} \mid\right. & \left.q^{A}\right)=U_{1}\left(A, \sigma_{2}, \sigma_{3} \mid q^{B}\right)= \\
& \begin{cases}\frac{1-\pi}{2}\left(6 \pi^{2}+5 \pi(1-\pi)+2(1-\pi)^{2}\right) & \text { if } \pi>3 / 4 \\
(1-\pi)\left(3 \pi^{2}+3 \pi(1-\pi)+(1-\pi)^{2}\right) & \text { if } 3 / 4>\pi>\frac{\sqrt[3]{3}}{1+\sqrt[3]{3}} \\
\frac{3(1-\pi)}{2}\left(2 \pi^{2}+2 \pi(1-\pi)+(1-\pi)^{2}\right) & \text { if } \pi<\frac{\sqrt[3]{3}}{1+\sqrt[3]{3}} .\end{cases}
\end{aligned}
$$

We have exactly $U_{1}\left(H, \sigma_{2}, \sigma_{3} \mid q^{H}\right) \geq U_{1}\left(\bar{H}, \sigma_{2}, \sigma_{3} \mid q^{H}\right)$ if and only if $\pi \geq 1 / 2$. Hence, the first bettor always follows his own private signal. This completes the proof.

\section{References}

Avery, C. And P. Zemsky (1998): "Multidimensional Uncertainty and Herd Behavior in Financial Markets," American Economic Review, 88, 724-748.

BAnerJee, A. (1992): "A Simple Model of Herd Behavior," Quarterly Journal of Economics, 107, 797-818.

Bikhchandani, S., D. Hirshleifer, and I. Welch (1992): "A Theory of Fads, Fashion, Custom, and Cultural Change as Informational Cascades," Journal of Political Economy, 100, 992-1026.

Brunnermeier, M. K. (2001): Asset Pricing under Asymmetric Information, Oxford University Press.

Chadha, S. And R. E. Quandt (1996): "Betting Bias and Market Equilibrium in Racetrack Betting," Applied Financial Economics, 6, 287-292.

Chamley, C. And D. Gale (1994): "Information Revelation and Strategic Delay in a Model of Investment," Econometrica, 62, 1065-1085.

Corsetti, G., A. Dasgupta, S. Morris, and H. S. Shin (2001): "Does One Soros Make a Difference? A Theory of Currency Crises with Large and Small Traders," mimeo, Yale University.

Dasgupta, A. (2000): "Social Learning with Payoff Complementarities," mimeo, Yale University.

Dekel, E. And M. Piccione (2000): "Sequential Voting Procedures in Symmetric Binary Elections," Journal of Political Economy, 108, 34-55.

Feddersen, T. And W. Pesendorfer (1998): "Convicting the Innocent: The Inferiority of Unanimous Jury Verdicts," American Political Science Review, 92, 23-35.

Feenay, R. And S. P. King (2001): "Sequential Parimutuel Games," Economics Letters, $72,165-173$.

HARsAnyi, J. C. (1967-1968): "Games with Incomplete Information Played by Bayesian Players. Parts I, II, III," Management Science, 14, 159-182, 320-334, 486-502.

Hurley, W. And L. MCDonough (1995): "A Note on the Hayek Hypothesis and the Favorite-Longshot Bias in Parimutuel Betting," American Economic Review, 85, 949-955. 
Koessler, F., A. Ziegelmeyer, and M.-H. Broihanne (2002): "The Favorite-Longshot Bias in Sequential Parimutuel Betting with Non-Expected Utility Players," Working Paper 2002-12, BETA, Université Louis Pasteur, Strasbourg.

Kreps, D. M. And R. Wilson (1982): "Sequential Equilibria," Econometrica, 50, 863-894.

SAUer, R. D. (1998): "The Economics of Wagering Markets," Journal of Economic Literature, 36, 2021-2064.

Terrell, D. And A. FArmer (1996): "Optimal Betting and Efficiency in Parimutuel Betting Markets with Information Costs," Economic Journal, 106, 846-868.

Vaughan Williams, L. (1999): "Information Efficiency in Betting Markets: A Survey," Bulletin of Economic Research, 51, 1-30.

Watanabe, T. (1997): "A Parimutuel System with two Horses and a Continuum of Bettors," Journal of Mathematical Economics, 28, 85-100.

Watanabe, T., H. Nonoyama, and M. Mori (1994): "A Model of a General Parimutuel System: Characterizations and Equilibrium Selection," International Journal of Game Theory, 23, 237-260.

Würtz, A. (1997): "A Universal Upper Bound on Power Functions," UNSW Discussion Paper 97/17.

Ziegelmeyer, A., M.-H. Broihanne, and F. Koessler (2001): "Sequential Parimutuel Betting in the Laboratory," mimeo, Université Louis Pasteur, Strasbourg. 